COMMUNICATIONS IN

ANALYSIS AND GEOMETRY

Volume 14, Number 4, 673-701, 2006

\title{
An explicit formula for the Webster pseudo-Ricci curvature on real hypersurfaces and its application for characterizing balls in $\mathbb{C}^{n}$
}

\author{
SONG-Ying Li AND Hing-Sun LuK
}

\begin{abstract}
In the paper, we provide an explicit formula for computing the Webster pseudo Ricci curvature, we also apply this formula to obtain a theorem on characterizing balls by using area and pseudo scalar curvature.
\end{abstract}

\section{Introduction and main results}

Let $M$ be a $(2 n+1)$-dimensional $\mathrm{CR}$ manifold with $\mathrm{CR}$ dimension $n$. We say that $(M, \theta)$ is a strictly pseudo-convex pseudo-Hermitian manifold in the sense of Webster [29] if $\theta$ is a real one-form (contact form) on $M$, and at each point of $M$, there is a neighborhood with a local basis $\theta^{1}, \ldots, \theta^{n}$ for the holomorphic cotangent space $T^{1,0}$ so that

$$
d \theta=i h_{\alpha \bar{\beta}} \theta^{\alpha} \wedge \theta^{\bar{\beta}}
$$

where $\left[h_{\alpha \bar{\beta}}\right]$ is a positive definite $n \times n$ matrix, determined by the Levi-form $L_{\theta}$ on $M$. Here,

$$
L_{\theta}(w, \bar{v})=-i d \theta(w, \bar{v}), \quad w, v \in T_{1,0}(M) .
$$

Let $R_{\alpha \bar{\beta}}$ be the Webster pseudo-Ricci curvature and let $R=h^{\alpha \bar{\beta}} R_{\alpha \bar{\beta}}$ be the pseudo-scalar curvature. It is known that the contact form $\theta$ is neither unique nor CR invariant, but lies in a conformal class $\left(\theta_{f}=\mathrm{e}^{f} \theta\right.$ for some smooth function $f$ ). There are many fundamental works done on CR manifolds by different authors. We refer to the book of Baouendi et al. [1], Beals et al. [2], Chang and Li [5], Chen and Shaw [6], Folland and Stein [10], Huang [14], Webster [28,29] and several papers of Jerison and Lee which will be mentioned later on. Here, we will address a few major problems on the Webster pseudo-Ricci curvature, which are related to the problems we are interested in this paper. 


\section{The CR Yamabe problem}

Find a contact form $\theta_{f}$ so that the Webster pseudo-scalar curvature $R_{\theta_{f}}$ with respect to $\theta_{f}$ is a given constant. The problem can be solved if the variational equation

$$
\lambda(M)=\inf _{\theta} \lambda(\theta)=\inf _{\theta} \frac{\int_{M} R_{\theta} \theta \wedge(d \theta)^{n}}{\left(\int_{M} \theta \wedge(d \theta)^{n}\right)^{n /(n+1)}},
$$

has a minimum. Much fundamental work has been done on the problem by Jerison and Lee in $[16,17,18]$, Gamara and Yacoub [11, 12]. In [16], Jerison and Lee proved that $\lambda(M) \leq \lambda\left(S^{2 n+1}\right)$ and solved the CR Yamabe problem for those $M$ with $\lambda(M)<\lambda\left(S^{2 n+1}\right)=n(n+1)$. In particular, they proved in [16] that if $\lambda(M)=\lambda\left(S^{2 n+1}\right)$, then $M$ is locally spherical. It suffices to consider $M=\partial D$, where $D$ is a smoothly bounded strictly pseudo-convex domain in $\mathbb{C}^{n+1}$. In addition, it was proved by Chern and Ji [8] that if $D$ is simply connected and local spherical then $D$ must global spherical, or $D$ is biholomorphical to the unit ball in $\mathbb{C}^{n+1}$. In this case, one can easily construct a contact form $\theta$ with constant pseudo-scalar curvature (see formula in Theorem 1.1 below). It was proved by Huang and Ji in [15] that if $M=\partial D$ is locally spherical, then $D$ is biholomorphically equivalent to the unit ball when $M$ is algebraic; and a counterexample was constructed by Burns and Schnider [3] that the algebraic condition cannot be replaced by real analyticity. For the general case when $\lambda(M)=\lambda\left(S^{2 n+1}\right)$, the CR Yamabe problem was solved by Gamara and Yacoub [12] and Gamara [11]. When $M=S^{2 n+1}$, the contact form $\theta$ with $\lambda(\theta)=n(n+1)$ was characterized by Jerison and Lee [17].

\section{The existence of pseudo-Hermitian Einstein metric}

$(M, \theta)$ is said to be pseudo-Einstein if

$$
R_{\alpha \bar{\beta}}=\left(\frac{1}{n}\right) R h_{\alpha \bar{\beta}}
$$

This problem was solved by Lee in his series of excellent works [20,21], where he gave a few characterizations for such manifolds. In particular, if there is a non-vanishing holomorphic $(n+1)$-form on $M$, then $M$ is pseudo-Einstein, which includes the boundary of strictly pseudo-convex domain in $\mathbb{C}^{n+1}$. In fact, the last result was obtained earlier by Luk in [27]. He showed that the boundary of any smoothly bounded strictly pseudo-convex domain 
$D=\{\rho(z)<0\}$ with the contact form $\theta=(-i / 2)(\partial \rho-\bar{\partial} \rho)$ generated by the potential function $\rho$ of the Fefferman metric is pseudo-Einstein.

Let $u \in C^{2}(D)$, and let $H(u)$ denote the complex hessian matrix of $u$. For any positive function $f(z) \in C^{\infty}(\bar{D})$. The boundary value problem for the Fefferman equation:

$$
J(\rho)=-\operatorname{det}\left[\begin{array}{cc}
\rho & \rho_{\bar{j}} \\
\rho_{i} & \rho_{i \bar{j}}
\end{array}\right] \equiv f(z) \text { in } D, \quad \rho=0 \text { on } \partial D,
$$

and with $U=-\log (-\rho)$ being strictly plurisubharmonic in $D$ has been studied by many mathematicians. When $D$ is a smoothly bounded strictly pseudo-convex domain in $\mathbb{C}^{n}$, the formal existence of such a solution was first given by Fefferman in [9] when $f \equiv 1$ on $D$; existence and uniqueness was proved by Cheng and Yau in [7] with $\rho \in C^{n+1+3 / 2}(\bar{D})$ for $f \in C^{\infty}(\bar{D})$. Lee and Melrose in [22] gave an asymptotic expansion for $\rho$; in particular, they showed $\rho \in C^{n+3-\epsilon}(\bar{D})$ for any $\epsilon>0$.

\section{Main results}

The best lower bound for the first positive eigenvalue of the sub-Laplace $\Delta_{b}=\operatorname{Re}\left(\square_{b}\right)$ where $\square_{b}$, is Kohn's Laplacian acting on functions, was given by Greenleaf [13] and $\mathrm{Li}$ and Luk in [26]. In the study of the eigenvalue problems, computability of the Webster pseudo-Ricci curvature is very important. This leads us to one of the main purposes of the present paper: to give an explicit formula for the Webster pseudo-Ricci curvatures and pseudoscalar curvature of $\partial D$ for a major class of contact forms. In other words, we will prove the following theorem.

Theorem 1.1. Let $M$ be a smooth strictly pseudo-convex hypersurface in $\mathbb{C}^{n+1}$. Let $U$ be a neighborhood of $M$, and let $\theta=(-i / 2)(\partial \rho-\bar{\partial} \rho)$, where $\rho \in C^{3}(U)$ is a defining function for $M$ with $J(\rho)>0$ in $U$. Then, for any $w, v \in T_{1,0}(M)$, the Webster pseudo-Ricci curvature is given by the formula:

$$
\operatorname{Ric}(w, \bar{v})=-\sum_{k, j=1}^{n+1} \frac{\partial^{2} \log J(\rho)}{\partial z^{k} \partial \bar{z}^{j}} w^{k} \bar{v}^{j}+(n+1) \frac{\operatorname{det} H(\rho)}{J(\rho)} L_{\theta}(w, \bar{v}) .
$$

In particular, if $\log J(\rho)$ is pluriharmonic near $\partial D$, then

$$
\operatorname{Ric}(w, \bar{v})=(n+1) \frac{\operatorname{det} H(\rho)}{J(\rho)} L_{\theta}(w, \bar{v}) \quad \text { and } \quad R=n(n+1) \frac{\operatorname{det} H(\rho)}{J(\rho)} .
$$


The characterization for balls in $\mathbb{C}^{n+1}$ is always an interesting subject $[8,15,19,23,29]$. Formula (1.7) in Theorem 1.1 and the main theorem in [23] on characterizing $D$ to be a ball in $\mathbb{C}^{n+1}$ lead us to the second main purpose of this paper by using the pseudo-scalar curvature to characterize a strictly pseudo-convex domain to be a ball. In order to state our result, we let $\rho_{0}$ be the unique plurisubharmoic solution of

$$
\operatorname{det} H\left(\rho_{0}\right)=J(\rho) \quad \text { in } D, \quad \rho_{0}(z)=0 \quad \text { on } \partial D \text {, }
$$

and let $\theta_{0}=1 / 2 i\left(\partial \rho_{0}-\bar{\partial} \rho_{0}\right)$. The existence and uinqueness of such a smooth $\rho_{0}$ was given by Caffarelli et al. in [4]. We denote the areas of $\partial D$ with respect to $\theta$ and $\theta_{0}$ as $\operatorname{Area}_{\theta}(\partial D)\left(:=\int_{\partial D} \theta \wedge(d \theta)^{n}\right)$ and $\operatorname{Area}_{\theta_{0}}(\partial D)$ $\left(:=\int_{\partial D} \theta_{0} \wedge\left(d \theta_{0}\right)^{n}\right)$. Let us first look at the case when $D$ is biholomorphic to a ball. If $\phi: D \rightarrow B_{n+1}$ is a biholomorphic mapping, then $\rho(z)=$ $|\phi(z)|^{2}-1$ solves the boundary value problem (1.5) with $J(\rho)=\left|\operatorname{det} \phi^{\prime}(z)\right|^{2}$ and $\log J(\rho)$ being pluriharmonic in $D$. In this case, the solution $\rho_{0}$ of $(1.8)$ agrees with $\rho$. Moreover, using formula (1.6), one can compute easily that the pseudo-scalar curvature $R_{\theta}=n(n+1)$, where $\theta=1 / 2 i(\partial \rho-\bar{\partial} \rho)$. Thus, we have

$$
\frac{R_{\theta}}{\operatorname{Area}_{\theta}(\partial D)}=\frac{n(n+1)}{\operatorname{Area}_{\theta_{0}}(\partial D)}
$$

Conversely, we have the following theorem.

Theorem 1.2. Let $D$ be a bounded strictly pseudo-convex domain in $\mathbb{C}^{n+1}$ with smooth boundary. Let $\rho \in C^{3}(\bar{D})$ be a defining function for $D$ with $J(\rho) \in C^{2}(\bar{D})$ being positive and $\log J(\rho)$ being pluriharmonic in $D$. Let $M=\partial D$ and $\theta=(-i / 2)(\partial \rho-\bar{\partial} \rho)$. Let $\theta_{0}=1 / 2 i\left(\partial \rho_{0}-\bar{\partial} \rho_{0}\right)$, with $\rho_{0}$ being the unique plurisubharmonic solution for (1.8). Then

(i) If the Webster pseudo-scalar curvature $R_{\theta}$ satisfies

$$
\frac{R_{\theta}}{\operatorname{Area}_{\theta}(\partial D)} \geq \frac{n(n+1)}{\operatorname{Area}_{\theta_{0}}(\partial D)}
$$

then $D$ must be biholomorphically equivalent to the unit ball in $\mathbb{C}^{n+1}$.

(ii) If $J(\rho) \equiv 1$ on $D$ and the Webster pseudo-scalar curvature $R_{\theta}$ satisfies (1.10), then $D$ must be biholomorphically equivalent to the unit ball in $\mathbb{C}^{n+1}$ under a constant Jacobian biholomorphic map. 
The paper is organized as follows. In Section 2, we will prove Theorem 1.1. Moreover, we provide some details for computing quantities related to the Webster pseudo-Ricci curvature of a general contact form. The proof of Theorem 1.2 will be given in Section 3.

\section{Pseudo-Ricci curvatures on hypersurfaces}

Let $M$ be a smooth hypersurface (of real dimension $(2 n+1)$ ) in $\mathbb{C}^{n+1}$. Let $\rho \in C^{2}\left(\mathbb{C}^{n+1}\right)$ be a defining function for $M: M=\{\rho=0\}$ and $\partial \rho \neq 0$ on $M$. Let $D=\left\{z \in \mathbb{C}^{n+1}: \rho(z)<0\right\}$. Let $u$ be a $C^{2}$ function on $D$, and we let $H(u)=\left[\partial^{2} u / \partial z^{i} \partial \bar{z}^{j}\right]_{(n+1) \times(n+1)}$ be the complex Hessian matrix of $u$. We use some notations in [24] and [25]. If $H(u)$ is invertible, then we let $\left[u^{k \bar{j}}\right]$ be the inverse of $H(u)^{t}$ so that

$$
\sum_{j=1}^{n+1} u^{k \bar{j}} u_{p \bar{j}}=\sum_{k=1}^{n+1} u^{\ell \bar{k}} u_{\ell \bar{p}}=\delta_{k p}
$$

Let $u_{k}=\partial u / \partial z^{k}, u_{\bar{j}}=\partial u / \partial \bar{z}^{j}$ and

$$
|\partial u|_{u}^{2}=u^{k \bar{j}} u_{k} u_{\bar{j}}, \quad u^{j}=\sum_{k=1}^{n+1} u^{j \bar{k}} u_{\bar{k}} \quad \text { and } \quad u^{\bar{j}}=\sum_{k=1}^{n+1} u^{k \bar{j}} u_{k} .
$$

Lemma 2.1. Let $D$ be a strictly pseudo-convex domain in $\mathbb{C}^{n+1}$ with smooth boundary $M$. Let $\rho$ be a smooth defining function for $D$ so that $H(\rho)$ is positive definite on $M$. Let

$$
\theta=\frac{1}{2 i}[\partial \rho-\bar{\partial} \rho], \quad \theta^{k}=d z^{k}-\frac{\rho^{k}}{|\partial \rho|_{\rho}^{2}} \partial \rho=d z^{k}-h^{k} \partial \rho .
$$

Then,

$$
\theta=-i \partial \rho=i \bar{\partial} \rho, \quad d \theta=i \sum_{k, \ell=1}^{n+1} \rho_{k \bar{\ell}} \theta^{k} \wedge \theta^{\bar{\ell}}
$$

Proof. Since

$$
\rho_{j} h^{j}=\frac{\rho_{j} \rho^{j}}{|\partial \rho|_{\rho}^{2}}=\frac{\rho_{j} \rho^{j \bar{k}} \rho_{\bar{k}}}{|\partial \rho|_{\rho}^{2}}=1, \quad \sum_{k=1}^{n+1} \rho_{k} \theta^{k}=\partial \rho-\partial \rho=0 \quad \text { on } M .
$$


Notice that since $d \rho=0$ on $\partial D$, we have $\partial \rho=-\bar{\partial} \rho$ and $\theta=-i \partial \rho=i \bar{\partial} \rho$ on $M$. Therefore,

$$
\begin{aligned}
d \theta & =i \partial \bar{\partial} \rho(z) \\
& =i \sum_{j, k=1}^{n+1} \rho_{k \bar{j}} d z^{k} \wedge d \bar{z}^{j} \\
& =i \sum_{j, k=1}^{n+1} \rho_{k \bar{j}} \theta^{k} \wedge d \bar{z}^{j}+i \sum_{j, k=1}^{n+1} \rho_{k \bar{j}} h^{k} \partial \rho \wedge d \bar{z}^{j} \\
& =i \sum_{j, k=1}^{n+1} \rho_{k \bar{j}} \theta^{k} \wedge \theta^{\bar{j}}+i \sum_{j, k=1}^{n+1} \rho_{k \bar{j}} h^{\bar{j}} \theta^{k} \wedge \bar{\partial} \rho+i \sum_{j, k=1}^{n+1} \rho_{k \bar{j}} h^{k} \partial \rho \wedge \theta^{\bar{j}}
\end{aligned}
$$

Then on $M$

$$
\begin{aligned}
\sum_{k=1}^{n+1} \sum_{j=1}^{n+1} \rho_{k \bar{j}} h^{\bar{j}} \theta^{k} & =\frac{1}{|\partial \rho|^{2}} \sum_{k=1}^{n+1} \sum_{j=1}^{n+1} \rho_{k \bar{j}} \sum_{\ell=1}^{n+1} \rho^{\ell^{\bar{j}}} \rho_{\ell} \theta^{k} \\
& =\frac{1}{|\partial \rho|^{2}} \sum_{k=1}^{n+1} \sum_{\ell=1}^{n+1} \delta_{k \ell} \rho_{\ell} \theta^{k} \\
& =\frac{\sum_{k=1}^{n+1} \rho_{k} \theta^{k}}{|\partial \rho|_{\rho}^{2}} \\
& =0 .
\end{aligned}
$$

We have proved that

$$
d \theta=i \sum_{j, k=1}^{n+1} \rho_{k \bar{j}} \theta^{k} \wedge \theta^{\bar{j}}
$$

and the proof of the lemma follows.

Let $M_{1}=\left\{z \in M: \rho_{n+1}(z) \neq 0\right\}$. Since

$$
\sum_{j=1}^{n+1} \rho_{j} \theta^{j}=0
$$

we can write

$$
\theta^{n+1}=-\sum_{k=1}^{n} \frac{\rho_{k}}{\rho_{n+1}} \theta^{k}
$$


Then,

$$
\begin{aligned}
d \theta & =i \sum_{j, k=1}^{n}\left[\rho_{k \bar{j}}-\frac{\rho_{n+1 \bar{j}} \rho_{k}}{\rho_{n+1}}-\frac{\rho_{k \overline{n+1}} \rho_{\bar{j}}}{\rho_{\overline{n+1}}}+\rho_{n+1} \overline{n+1} \frac{\rho_{k} \rho_{\bar{j}}}{\left|\rho_{n+1}\right|^{2}}\right] \theta^{k} \wedge \theta^{\bar{j}} \\
& :=i \sum_{\alpha, \beta=1}^{n} h_{\alpha \bar{\beta}} \theta^{\alpha} \wedge \theta^{\bar{\beta}} .
\end{aligned}
$$

Let

$$
Y_{j}=\frac{\partial}{\partial z^{j}}-\frac{\rho_{j}}{\rho_{n+1}} \frac{\partial}{\partial z^{n+1}}, \quad Y=i \sum_{j=1}^{n+1}\left(h^{j} \frac{\partial}{\partial z^{j}}-h^{\bar{j}} \frac{\partial}{\partial \bar{z}^{j}}\right)
$$

Then,

$$
\begin{aligned}
\sum_{p=1}^{n} h_{\beta \bar{p}} h^{\bar{p}}= & \sum_{p=1}^{n}\left[\rho_{\beta \bar{p}}-\frac{\rho_{n+1 \bar{p}} \rho_{\beta}}{\rho_{n+1}}-\frac{\rho_{\beta \overline{n+1}} \rho_{\bar{p}}}{\rho_{\overline{n+1}}}+\rho_{n+1 \overline{n+1}} \frac{\rho_{\beta} \rho_{\bar{p}}}{\left|\rho_{n+1}\right|^{2}}\right] h^{\bar{p}} \\
= & \frac{\rho_{\beta}}{|\partial \rho|_{\rho}^{2}}-\rho_{\beta \overline{n+1}} h^{\overline{n+1}}-\frac{\rho_{\beta}}{|\partial|_{\rho}^{2}}+\frac{\rho_{n+1} \overline{n+1} \rho_{\beta} h^{\overline{n+1}}}{\rho_{n+1}} \\
& +\sum_{p=1}^{n}\left[-\frac{\rho_{\beta \overline{n+1}}}{\rho_{\overline{n+1}}}+\rho_{n+1 \overline{n+1}} \frac{\rho_{\beta}}{\left|\rho_{n+1}\right|^{2}}\right] \rho_{\bar{p}} h^{\bar{p}} \\
= & -\rho_{\beta \overline{n+1}} h^{\overline{n+1}}+\frac{\rho_{n+1} \overline{n+1} \rho_{\beta} h^{\overline{n+1}}}{\rho_{n+1}} \\
& +\left[-\frac{\rho_{\beta \overline{n+1}} \rho_{\beta}}{\rho_{\overline{n+1}}}+\rho_{n+1 \overline{n+1}} \frac{\rho_{\beta}}{\left|\rho_{n+1}\right|^{2}}\right]\left(1-\rho_{\overline{n+1}} h^{\overline{n+1}}\right) \\
= & -\frac{\rho_{\beta \overline{n+1}}}{\rho_{\overline{n+1}}}+\rho_{n+1} \overline{\rho_{\beta}} \\
= & -Y_{\beta} \log \rho_{\overline{n+1}} .
\end{aligned}
$$

Moreover, for any $f \in C^{1}\left(M_{1}\right)$, a standard computation [29] shows that

$$
d f=\sum_{j=1}^{n+1} f_{j} d z^{j}+f_{\bar{j}} d \bar{z}^{j}=\sum_{j=1}^{n}\left(Y_{j} f \theta^{j}+Y_{\bar{j}} f \theta^{\bar{j}}\right)+Y(f) \theta
$$


Notice that $\theta^{\alpha}=d z^{\alpha}-i h^{\alpha} \theta$. We have

$$
\begin{aligned}
d \theta^{\alpha} & =-i d h^{\alpha} \wedge \theta-i h^{\alpha} d \theta \\
& =-i d h^{\alpha} \wedge \theta+h^{\alpha} h_{\gamma \bar{\beta}} \theta^{\gamma} \wedge \theta^{\bar{\beta}} \\
& =\sum_{\gamma=1}^{n} \theta^{\gamma} \wedge\left(h^{\alpha} \sum_{\beta=1}^{n} h_{\gamma \bar{\beta}^{\beta}} \theta^{\bar{\beta}}-i Y_{\gamma} h^{\alpha} \theta\right)+\theta \wedge\left(\sum_{\beta=1}^{n} i \bar{Y}_{\beta} h^{\alpha} \theta^{\bar{\beta}}\right) \\
& =\sum_{\gamma=1}^{n} \theta^{\gamma} \wedge \omega_{\gamma}^{\alpha}+\theta \wedge \tau^{\alpha},
\end{aligned}
$$

where

$$
\omega_{\gamma}^{\prime \alpha}=h^{\alpha} \sum_{\beta=1}^{n} h_{\gamma \bar{\beta}} \theta^{\bar{\beta}}-i Y_{\gamma} h^{\alpha} \theta, \quad \tau^{\alpha}=i \sum_{\beta=1}^{n} Y_{\bar{\beta}} h^{\alpha} \theta^{\bar{\beta}}
$$

and

$$
\tau_{\alpha}=h_{\alpha \bar{\gamma}} \tau^{\bar{\gamma}}=-i \sum_{\beta=1}^{n} \sum_{\gamma=1}^{n} h_{\alpha \bar{\gamma}} Y_{\beta} h^{\bar{\gamma}} \theta^{\beta}=\sum_{\beta=1}^{n} \mathcal{A}_{\alpha \beta} \theta^{\beta}
$$

with

$$
\mathcal{A}_{\alpha \beta}=(-i) \sum_{\gamma=1}^{n} h_{\alpha \bar{\gamma}} Y_{\beta} h^{\bar{\gamma}}
$$

The torsion of $M$ with respect to $\theta$ (see (1.20) in Webster [28]) is defined as follows:

$$
\operatorname{Tor}\left(z^{\alpha} Y_{\alpha}, w^{\beta} Y_{\beta}\right)=i\left(\mathcal{A}_{\bar{\alpha} \bar{\beta}} z^{\bar{\alpha}} w^{\bar{\beta}}-\mathcal{A}_{\alpha \beta} z^{\alpha} w^{\beta}\right) .
$$

Using the fact $d \theta=i h_{\alpha \bar{\beta}} \theta^{\alpha} \wedge \theta^{\bar{\beta}}$ on $M_{1}$, one has

$$
\begin{aligned}
0 & =d d \theta \\
& =d h_{\alpha \bar{\beta}} \wedge \theta^{\alpha} \wedge \theta^{\bar{\beta}}+h_{\alpha \bar{\beta}} d \theta^{\alpha} \wedge \theta^{\bar{\beta}}-h_{\alpha \bar{\beta}} \theta^{\alpha} \wedge d \theta^{\bar{\beta}} \\
& =d h_{\alpha \bar{\beta}} \wedge \theta^{\alpha} \wedge \theta^{\bar{\beta}}+h_{\alpha \bar{\beta}}\left[\left(\theta^{\gamma} \wedge \omega_{\gamma}^{\prime \alpha}+\theta \tau^{\alpha}\right) \wedge \theta^{\bar{\beta}}-\theta^{\alpha} \wedge\left(\theta^{\bar{\gamma}} \wedge \omega^{\prime \bar{\beta}}+\theta \wedge \tau^{\bar{\beta}}\right)\right] \\
& =\left(d h_{\alpha \bar{\beta}}-h_{\gamma \bar{\beta}} \omega_{\alpha}^{\prime \gamma}-h_{\alpha \bar{\gamma}} \omega_{\bar{\beta}}^{\prime \bar{\gamma}}\right) \theta^{\alpha} \wedge \theta^{\bar{\beta}}+\theta \wedge \tau_{\bar{\beta}} \wedge \theta^{\bar{\beta}}-\theta \wedge \tau_{\alpha} \wedge \theta^{\alpha} .
\end{aligned}
$$

This implies that

$$
\tau_{\alpha} \wedge \theta^{\alpha}=0 \quad \text { or } \quad \mathcal{A}_{\alpha \beta}=\mathcal{A}_{\beta \alpha}
$$


and

$$
d h_{\alpha \bar{\beta}}-\omega_{\alpha \bar{\beta}}^{\prime}-\omega_{\bar{\beta} \alpha}^{\prime}=d h_{\alpha \bar{\beta}}-h_{\gamma \bar{\beta}} \omega_{\alpha}^{\prime \gamma}-h_{\alpha \bar{\gamma}} \omega^{\prime \bar{\gamma}}=\mathcal{A}_{\alpha \bar{\beta} \gamma} \theta^{\gamma}+\mathcal{B}_{\alpha \bar{\beta} \bar{\gamma}} \theta^{\bar{\gamma}}
$$

where

$$
\mathcal{A}_{\alpha \bar{\beta} \gamma}=\mathcal{A}_{\bar{\gamma} \bar{\beta} \alpha}, \quad \mathcal{B}_{\alpha \bar{\beta} \bar{\gamma}}=\mathcal{A}_{\bar{\beta} \alpha \bar{\gamma}}
$$

Then,

$$
\begin{aligned}
\omega_{\alpha \bar{\beta}}^{\prime} & =h_{\gamma \bar{\beta}} \omega_{\alpha}^{\prime \gamma} \\
& =h_{\gamma \bar{\beta}}\left(h^{\gamma} \sum_{k=1}^{n} h_{\alpha \bar{k}} \theta^{\bar{k}}-i Y_{\alpha} h^{\gamma} \theta\right) \\
& =h_{\bar{\beta}} \sum_{k=1}^{n} h_{\alpha \bar{k}} \theta^{\bar{k}}-i h_{\gamma \bar{\beta}} Y_{\alpha} h^{\gamma} \theta
\end{aligned}
$$

and

$$
\mathcal{A}_{\alpha \bar{\beta} \gamma}=Y_{\gamma} h_{\alpha \bar{\beta}}-h_{\alpha} h_{\gamma \bar{\beta}}
$$

If we let

$$
\begin{aligned}
\omega_{\alpha \bar{\beta}} & =\omega_{\alpha \bar{\beta}}^{\prime}+\left(Y_{\gamma} h_{\alpha \bar{\beta}}-h_{\alpha} h_{\gamma \bar{\beta}}\right) \theta^{\gamma} \\
& =\left(Y_{\gamma} h_{\alpha \bar{\beta}}-h_{\alpha} h_{\gamma \bar{\beta}}\right) \theta^{\gamma}+h_{\bar{\beta}} h_{\alpha \bar{\gamma}} \theta^{\bar{\gamma}}-i h_{\gamma \bar{\beta}} Y_{\alpha} h^{\gamma} \theta
\end{aligned}
$$

and

$$
\omega_{\bar{\beta} \alpha}=\left(Y_{\bar{\gamma}} h_{\alpha \bar{\beta}}-h_{\bar{\beta}} h_{\alpha \bar{\gamma}}\right) \theta^{\bar{\gamma}}+h_{\alpha} h_{\gamma \bar{\beta}} \theta^{\gamma}+i h_{\alpha \bar{\gamma}} Y_{\bar{\beta}} h^{\bar{\gamma}} \theta
$$

then

$$
d h_{\alpha \bar{\beta}}-h_{\gamma \bar{\beta}} \omega_{\alpha}^{\gamma}-h_{\alpha \bar{\gamma}} \omega_{\bar{\beta}}^{\bar{\gamma}}=0
$$

and

$$
Y_{\gamma} h_{\alpha \bar{\beta}}-h_{\alpha} h_{\gamma \bar{\beta}}=Y_{\alpha} h_{\gamma \bar{\beta}}-h_{\gamma} h_{\alpha \bar{\beta}} .
$$

Note. We should note that the purpose of changing $\omega_{\alpha}^{\prime \beta}$ in $(2.16)$ to $\omega_{\alpha}^{\beta}$ in (2.21) is to modify the connection so that it is compatible with the metric induced by the Levi form (1.2). 
Let

$$
\Omega_{\beta}^{\alpha}=d \omega_{\beta}^{\alpha}-\omega_{\beta}^{\gamma} \wedge \omega_{\gamma}^{\alpha}-i \theta_{\beta} \wedge \tau^{\alpha}+i \tau_{\beta} \wedge \theta^{\alpha} .
$$

Then,

$$
\begin{aligned}
\Omega_{\alpha \bar{\beta}} & =h_{\gamma \bar{\beta}} \Omega_{\alpha}^{\gamma} \\
& =h_{\gamma \bar{\beta}}\left[d \omega_{\alpha}^{\gamma}-\omega_{\alpha}^{k} \wedge \omega_{k}^{\gamma}-i \theta_{\alpha} \wedge \tau^{\gamma}+i \tau_{\alpha} \wedge \theta^{\gamma}\right] \\
& =-d h_{\gamma \bar{\beta}} \wedge \omega_{\alpha}^{\gamma}+d \omega_{\alpha \bar{\beta}}-\omega_{\alpha}^{k} \wedge \omega_{k \bar{\beta}}-i \theta_{\alpha} \wedge \tau_{\bar{\beta}}+i \tau_{\alpha} \wedge \theta_{\bar{\beta}} \\
& =-\left[h_{k \bar{\beta}} \omega_{\gamma}^{k}+h_{\gamma \bar{k}} \omega_{\bar{\beta}}^{\bar{k}}\right] \wedge \omega_{\alpha}^{\gamma}+d \omega_{\alpha \bar{\beta}}-\omega_{\alpha}^{k} \wedge \omega_{k \bar{\beta}}-i \theta_{\alpha} \wedge \tau_{\bar{\beta}}+i \tau_{\alpha} \wedge \theta_{\bar{\beta}} \\
& =-\left[\omega_{\gamma \bar{\beta}}+\omega_{\bar{\beta} \gamma}\right] \wedge \omega_{\alpha}^{\gamma}+d \omega_{\alpha \bar{\beta}}-\omega_{\alpha}^{k} \wedge \omega_{k \bar{\beta}}-i \theta_{\alpha} \wedge \tau_{\bar{\beta}}+i \tau_{\alpha} \wedge \theta_{\bar{\beta}} \\
(2.24) & =d \omega_{\alpha \bar{\beta}}-\omega_{\bar{\beta} \gamma} \wedge \omega_{\alpha}^{\gamma}-i \theta_{\alpha} \wedge \tau_{\bar{\beta}}+i \tau_{\alpha} \wedge \theta_{\bar{\beta}} .
\end{aligned}
$$

We compute

$$
\begin{aligned}
d \omega_{\alpha \bar{\beta}} & \left(\bmod \theta, \theta^{p} \wedge \theta^{q}, \theta^{\bar{p}} \wedge \theta^{\bar{q}}\right) \\
= & {\left[-Y_{\bar{\ell}} Y_{\gamma} h_{\alpha \bar{\beta}}+Y_{\bar{\ell}}\left(h_{\alpha} h_{\gamma \bar{\beta}}\right)+Y_{\gamma}\left(h_{\bar{\beta}} h_{\alpha \bar{\ell}}\right)\right] \theta^{\gamma} \wedge \theta^{\bar{\ell}}+\left(Y_{\gamma} h_{\alpha \bar{\beta}}-h_{\alpha} h_{\gamma \bar{\beta}}\right) d \theta^{\gamma} } \\
& +h_{\bar{\beta}} h_{\alpha \bar{\gamma}} d \theta^{\bar{\gamma}}+h_{j \bar{\beta}} Y_{\alpha} h^{j} h_{\gamma \bar{\ell}} \theta^{\gamma} \wedge \theta^{\bar{\ell}} \\
= & {\left[-Y_{\bar{\ell}} Y_{\gamma} h_{\alpha \bar{\beta}}+Y_{\bar{\ell}}\left(h_{\alpha} h_{\gamma \bar{\beta}}\right)+Y_{\gamma}\left(h_{\bar{\beta}} h_{\alpha \bar{\ell}}\right)+h_{\gamma \bar{\ell}} h_{j \bar{\beta}} Y_{\alpha} h^{j}\right] \theta^{\gamma} \wedge \theta^{\bar{\ell}} } \\
& +\left(Y_{\gamma} h_{\alpha \bar{\beta}}-h_{\alpha} h_{\gamma \bar{\beta}}\right) h^{\gamma} \sum_{k=1}^{n} h_{j \bar{k}} \theta^{j} \wedge \theta^{\bar{k}}+h_{\bar{\beta}} h_{\alpha \bar{\gamma}} h^{\bar{\gamma}} \sum_{k=1}^{n} h_{k \bar{j}} \theta^{\bar{j}} \wedge \theta^{k} \\
= & {\left[-Y_{\bar{\ell}} Y_{\gamma} h_{\alpha \bar{\beta}}+Y_{\bar{\ell}}\left(h_{\alpha} h_{\gamma \bar{\beta}}\right)+Y_{\gamma}\left(h_{\bar{\beta}} h_{\alpha \bar{\ell}}\right)+h_{\gamma \bar{\ell}} h_{j \bar{\beta}} Y_{\alpha} h^{j}\right.} \\
& \left.+\left(Y_{j} h_{\alpha \bar{\beta}}-h_{\alpha} h_{j \bar{\beta}}\right) h^{j} h_{\gamma \bar{\ell}}-h_{\bar{\beta}} h_{\alpha \bar{k}} h^{\bar{k}} h_{\gamma \bar{\ell}}\right] \theta^{\gamma} \wedge \theta^{\bar{\ell}} \\
= & {\left[-Y_{\bar{\ell}} Y_{\gamma} h_{\alpha \bar{\beta}}+Y_{\bar{\ell}}\left(h_{\alpha} h_{\gamma \bar{\beta}}\right)+Y_{\gamma}\left(h_{\bar{\beta}} h_{\alpha \bar{\ell}}\right)+h_{\gamma \bar{\ell}} h_{j \bar{\beta}} Y_{\alpha} h^{j}\right.} \\
& \left.+h_{\gamma \bar{\ell}} h^{j} Y_{j} h_{\alpha \bar{\beta}}-2 h_{\bar{\beta}} h_{\alpha} h_{\gamma \bar{\ell}}\right] \theta^{\gamma} \wedge \theta^{\bar{\ell}}
\end{aligned}
$$

and

$$
\begin{aligned}
\omega_{\alpha}^{\gamma} & =h^{\gamma \bar{\ell}} \omega_{\alpha \bar{\ell}} \\
& =h^{\gamma \bar{\ell}}\left(Y_{j} h_{\alpha \bar{\ell}}-h_{\alpha} h_{j \bar{\ell}}\right) \theta^{j}+h^{\gamma \bar{\ell}} h_{\bar{\ell}} h_{\alpha \bar{j}} \theta^{\bar{j}} \bmod \theta \\
& =\left(h^{\gamma \bar{\ell}} Y_{j} h_{\alpha \bar{\ell}}-h_{\alpha} \delta_{j \gamma}\right) \theta^{j}+h^{\gamma} h_{\alpha \bar{j}} \theta^{\bar{j}} \bmod \theta .
\end{aligned}
$$

By (2.20), we have

$$
\omega_{\bar{\beta} \gamma}=\left(Y_{\bar{p}} h_{\gamma \bar{\beta}}-h_{\bar{\beta}} h_{p \bar{\gamma}}\right) \theta^{\bar{p}}+h_{\gamma} h_{p \bar{\beta}} \theta^{p} \bmod \theta .
$$


Thus,

$$
\begin{aligned}
& \omega_{\bar{\beta} \gamma} \wedge \omega_{\alpha}^{\gamma} \bmod \theta, \theta^{p} \wedge \theta^{q}, \theta^{\bar{p}} \wedge \theta^{\bar{q}} \\
&=\left[\left(Y_{\bar{p}} h_{\gamma \bar{\beta}}-h_{\bar{\beta}} h_{\gamma \bar{p}}\right) \theta^{\bar{p}}+h_{\gamma} h_{p \bar{\beta}} \theta^{p}\right] \wedge\left[\left(h^{\gamma \bar{\ell}} Y_{j} h_{\alpha \bar{\ell}}-h_{\alpha} \delta_{j \gamma}\right) \theta^{j}+h^{\gamma} h_{\alpha \bar{j}} \theta^{\bar{j}}\right] \\
&=h_{\gamma} h_{p \bar{\beta}} h^{\gamma} h_{\alpha \bar{j}} \theta^{p} \wedge \theta^{\bar{j}}-\left(Y_{\bar{p}} h_{\gamma \bar{\beta}}-h_{\bar{\beta}} h_{\gamma \bar{p}}\right)\left(h^{\gamma \bar{\ell}} Y_{j} h_{\alpha \bar{\ell}}-h_{\alpha} \delta_{j \gamma}\right) \theta^{j} \wedge \theta^{\bar{p}} \\
&=\left[h_{\gamma} h^{\gamma} h_{p \bar{\beta}} h_{\alpha \bar{j}}-\left(Y_{\bar{j}} h_{\gamma \bar{\beta}}-h_{\bar{\beta}} h_{\gamma \bar{j}}\right)\left(h^{\gamma \bar{\ell}} Y_{p} h_{\alpha \bar{\ell}}-h_{\alpha} \delta_{p \gamma}\right)\right] \theta^{p} \wedge \theta^{\bar{j}} \\
&=\left[h_{\gamma} h^{\gamma} h_{p \bar{\beta}} h_{\alpha \bar{j}}-h^{\gamma \bar{\ell}} Y_{j} h_{\gamma \bar{\beta}} Y_{p} h_{\alpha \bar{\ell}}+h_{\alpha} \delta_{p \gamma} Y_{\bar{j}} h_{\gamma \bar{\beta}}\right. \\
& \\
&\left.+h_{\bar{\beta}} h_{\gamma \bar{j}} h^{\gamma \bar{\ell}} Y_{p} h_{\alpha \bar{\ell}}-h_{\bar{\beta}} h_{\alpha} h_{\gamma \bar{j}} \delta_{p \gamma}\right] \theta^{p} \wedge \theta^{\bar{j}} \\
&=\left[h_{\gamma} h^{\gamma} h_{p \bar{\beta}} h_{\alpha \bar{j}}-h^{\gamma \bar{\ell}} Y_{\bar{j}} h_{\gamma \bar{\beta}} Y_{p} h_{\alpha \bar{\ell}}+h_{\alpha} Y_{\bar{j}} h_{p \bar{\beta}}+h_{\bar{\beta}} Y_{p} h_{\alpha \bar{j}}-h_{\bar{\beta}} h_{\alpha} h_{p \bar{j}} \theta^{p} \wedge \theta^{\bar{j}}\right. \\
&= {\left[h_{j} h^{j} h_{\gamma \bar{\beta}} h_{\alpha \bar{\ell}}-h^{j \bar{q}} Y_{\bar{\ell}} h_{j \bar{\beta}} Y_{\gamma} h_{\alpha \bar{q}}+h_{\alpha} Y_{\bar{\ell}} h_{\gamma \bar{\beta}}+h_{\bar{\beta}} Y_{\gamma} h_{\alpha \bar{\ell}}-h_{\bar{\beta}} h_{\alpha} h_{\gamma \bar{\ell}}\right] \theta^{\gamma} \wedge \theta^{\bar{\ell}} . }
\end{aligned}
$$

Therefore,

$$
\Omega_{\alpha \bar{\beta}}=R_{\alpha \bar{\beta} \gamma \bar{\ell}} \theta^{\gamma} \wedge \theta^{\bar{\ell}}+\theta \wedge \lambda_{\alpha \bar{\beta}}
$$

where

$$
\begin{aligned}
R_{\alpha \bar{\beta} \gamma \bar{\ell}}= & -Y_{\bar{\ell}} Y_{\gamma} h_{\alpha \bar{\beta}}+Y_{\bar{\ell}}\left(h_{\alpha} h_{\gamma \bar{\beta}}\right)+Y_{\gamma}\left(h_{\bar{\beta}} h_{\alpha \bar{\ell}}\right)+h_{\gamma \bar{\ell}} h_{j \bar{\beta}} Y_{\alpha} h^{j} \\
& +h_{\gamma \bar{\ell}} h^{j} Y_{j} h_{\alpha \bar{\beta}}-2 h_{\bar{\beta}} h_{\alpha} h_{\gamma \bar{\ell}}-h_{j} h^{j} h_{\gamma \bar{\beta}} h_{\alpha \bar{\ell}} \\
& +h^{j \bar{q}} Y_{\bar{\ell}} h_{j \bar{\beta}} Y_{\gamma} h_{\alpha \bar{q}}-h_{\alpha} Y_{\bar{\ell}} h_{\gamma \bar{\beta}}-h_{\bar{\beta}} Y_{\gamma} h_{\alpha \bar{\ell}}+h_{\bar{\beta}} h_{\alpha} h_{\gamma \bar{\ell}}
\end{aligned}
$$

Let

$$
g(z)=\log \operatorname{det}\left(h_{\alpha \bar{\beta}}\right), \quad z \in M_{1} .
$$

Then, for $1 \leq \gamma, \ell \leq n$, we have

$$
\begin{aligned}
R_{\gamma \bar{\ell}}= & h^{\alpha \bar{\beta}} R_{\alpha \bar{\beta} \gamma \bar{\ell}} \\
= & -h^{\alpha \bar{\beta}} Y_{\bar{\ell}} Y_{\gamma} h_{\alpha \bar{\beta}}+Y_{\bar{\ell}}\left(h_{\gamma}\right)+h^{\bar{\beta}} Y_{\bar{\ell}}\left(h_{\gamma \bar{\beta}}\right)+Y_{\gamma}\left(h_{\bar{\ell}}\right)+h^{\alpha} Y_{\gamma}\left(h_{\alpha \bar{\ell}}\right) \\
& +h_{\gamma \bar{\ell}} Y_{\alpha} h^{\alpha}+h_{\gamma \bar{\ell}} h^{j} Y_{j} g-2 h^{\alpha} h_{\alpha} h_{\gamma \bar{\ell}}-h_{j} h^{j} h_{\gamma \bar{\ell}}+h^{\alpha \bar{\beta}} h^{j \bar{q}} Y_{\bar{\ell}} h_{j \bar{\beta}} Y_{\gamma} h_{\alpha \bar{q}} \\
& -h^{\bar{\beta}} Y_{\bar{\ell}} h_{\gamma \bar{\beta}}-h^{\alpha} Y_{\gamma} h_{\alpha \bar{\ell}}+h^{\alpha} h_{\alpha} h_{\gamma \bar{\ell}} \\
= & -h^{\alpha \bar{\beta}} Y_{\bar{\ell}} Y_{\gamma} h_{\alpha \bar{\beta}}+h^{\alpha \bar{\beta}} h^{j \bar{q}} Y_{\bar{\ell}} h_{j \bar{\beta}} Y_{\gamma} h_{\alpha \bar{q}}+Y_{\bar{\ell}}\left(h_{\gamma}\right)+Y_{\gamma}\left(h_{\bar{\ell}}\right) \\
& +\left[Y_{\alpha} h^{\alpha}+h^{j} Y_{j} g-2 h^{\alpha} h_{\alpha}\right] h_{\gamma \bar{\ell}}
\end{aligned}
$$




$$
\begin{aligned}
= & -Y_{\bar{\ell}} Y_{\gamma} g(z)+Y_{\bar{\ell}}\left(h_{\gamma}\right)+Y_{\gamma}\left(h_{\bar{\ell}}\right)+\left[Y_{\alpha} h^{\alpha}+h^{j} Y_{j} g-2 h^{\alpha} h_{\alpha}\right] h_{\gamma \bar{\ell}} \\
= & -Y_{\bar{\ell}} Y_{\gamma} g(z)-Y_{\bar{\ell}} Y_{\gamma}\left(\log \rho_{\overline{n+1}}\right)-Y_{\gamma} Y_{\bar{\ell}}\left(\log \rho_{n+1}\right) \\
& +\left[Y_{\alpha} h^{\alpha}+h^{j} Y_{j} g-2 h^{\alpha} h_{\alpha}\right] h_{\bar{\gamma} \bar{\ell}} \\
= & -Y_{\bar{\ell}} Y_{\gamma} g(z)-Y_{\bar{\ell}} Y_{\gamma}\left(\log \left|\rho_{n+1}\right|^{2}\right)+\left[Y_{\bar{\ell}}, Y_{\gamma}\right]\left(\log \rho_{n+1}\right) \\
& +\left[Y_{\alpha} h^{\alpha}+h^{j} Y_{j} g-2 h^{\alpha} h_{\alpha}\right] h_{\gamma \bar{\ell}} .
\end{aligned}
$$

Since

$$
\left[Y_{\bar{\ell}}, Y_{\gamma}\right]=-Y_{\bar{\ell}}\left(\frac{\rho_{\gamma}}{\rho_{n+1}}\right) \partial_{n+1}+Y_{\gamma}\left(\frac{\rho_{\bar{\ell}}}{\rho_{\overline{n+1}}}\right) \partial_{\overline{n+1}}=h_{\gamma \bar{\ell}}\left[\frac{\partial_{\overline{n+1}}}{\rho_{\overline{n+1}}}-\frac{\partial_{n+1}}{\rho_{n+1}}\right],
$$

we get

$$
\left[Y_{\bar{\ell}}, Y_{\gamma}\right]\left(\log \rho_{n+1}\right)=\left(\frac{\rho_{n+1} \overline{n+1}}{\left|\rho_{n+1}\right|^{2}}-\frac{\rho_{n+1 n+1}}{\rho_{n+1}^{2}}\right) h_{\gamma \bar{\ell}} .
$$

Lemma 2.2. With the notation above, one has the following identity

$$
g(z)+\log \left|\rho_{n+1}\right|^{2}=\log \operatorname{det}\left[h_{\alpha \bar{\beta}}\right]+\log \left|\rho_{n+1}\right|^{2}=\log [J(\rho)+\rho \operatorname{det} H(\rho)] .
$$

Proof. Let $\bar{\partial} \rho=\left(\partial_{\overline{1}} \rho, \ldots, \partial_{\overline{n+1}} \rho\right)$ be viewed as a $1 \times(n+1)$ matrix. With the notation $|\partial \rho|_{\rho}^{2}=\sum_{i, j=1}^{n+1} \rho^{i \bar{j}} \rho_{i} \rho_{\bar{j}}$, we have

$$
\begin{aligned}
J(\rho) & =-\operatorname{det}\left[\begin{array}{cc}
\rho & \bar{\partial} \rho \\
(\bar{\partial} \rho)^{*} & H(\rho)
\end{array}\right] \\
& =-\rho \operatorname{det}\left(H(\rho)-\frac{1}{\rho}(\bar{\partial} \rho)^{*} \bar{\partial} \rho\right) \\
& =-\rho \operatorname{det} H(\rho)\left(1-\frac{1}{\rho}|\partial \rho|_{\rho}^{2}\right) .
\end{aligned}
$$

Therefore,

$$
J(\rho)+\rho \operatorname{det} H(\rho)=\operatorname{det} H(\rho)|\partial \rho|_{\rho}^{2} .
$$

For $z^{0} \in M_{1}$, we discuss it in two cases:

(a) if $\rho_{j}\left(z^{0}\right)=0$ for all $1 \leq j \leq n$, then

$$
\operatorname{det} H(\rho)|\partial \rho|_{\rho}^{2}\left(z_{0}\right)=\left|\rho_{n+1}\right|^{2} \operatorname{det}\left[\rho_{\alpha \bar{\beta}}\right]\left(z_{0}\right)=\left|\rho_{n+1}\right|^{2} \operatorname{det}\left[h_{\alpha \bar{\beta}}\right]\left(z_{0}\right),
$$

and (2.31) holds. 
(b) if $\rho_{j}\left(z^{0}\right) \neq 0$ for some $1 \leq j \leq n$, then we let

$z_{j}=w_{j}, \quad 1 \leq j \leq n ; \quad z_{n+1}=w_{n+1}-\sum_{\alpha=1}^{n} \frac{\rho_{\alpha}\left(z^{0}\right)}{\rho_{n+1}\left(z^{0}\right)} \sum_{j=1}^{n+1} \rho_{j}\left(z^{0}\right) w_{j}$

and let $r(w)=\rho(z(w))$ and $z^{0}=z\left(w^{0}\right)$. Since $\operatorname{det}(\partial z / \partial w)\left(w^{0}\right)=1$ and $\partial r / \partial w_{\alpha}\left(w^{0}\right)=0$ for all $1 \leq \alpha \leq n$, one can easily see by the previous case that

$\operatorname{det} H(\rho)|\partial \rho|_{\rho}^{2}\left(z^{0}\right)=\operatorname{det} H(r)|\partial r|_{r}^{2}\left(w^{0}\right)=\left|r_{n+1}\left(w^{0}\right)\right|^{2} \operatorname{det}\left[r_{\alpha \bar{\beta}}\right]\left(w^{0}\right)$

A simple computation shows that $\left|r_{n+1}\left(w^{0}\right)\right|^{2}=\left|\rho_{n+1}\left(z^{0}\right)\right|^{2}$ and for $1 \leq \alpha$, $\beta \leq n$

$$
\begin{aligned}
\frac{\partial^{2} r}{\partial w_{\alpha} \partial \bar{w}_{\beta}}\left(w^{0}\right) & =\rho_{\alpha \bar{\beta}}\left(z^{0}\right)-\frac{\rho_{n+1 \bar{\beta}} \rho_{\alpha}}{\rho_{n+1}}-\frac{\rho_{\alpha \overline{n+1}}}{\rho_{\overline{n+1}}} \rho_{\bar{\beta}}+\rho_{n+1} \overline{n+1} \\
\frac{\rho_{\alpha} \rho_{\bar{\beta}}}{\left|\rho_{n+1}\right|^{2}}\left(z^{0}\right) & =h_{\alpha \bar{\beta}}\left(z^{0}\right) .
\end{aligned}
$$

Therefore, $\operatorname{det} H(\rho)\left(z^{0}\right)|\partial \rho|_{\rho}^{2}\left(z^{0}\right)=\left|\rho_{n+1}\left(z^{0}\right)\right|^{2} \operatorname{det}\left[h_{\alpha \bar{\beta}}\left(z^{0}\right)\right]$, and the proof is complete.

Combining all the above, particularly $(2.31)$ and that $Y_{\bar{\ell}}, Y_{\gamma}$ are tangential to $M_{1}$, we have, on $M_{1}$

$$
\begin{aligned}
R_{\gamma \bar{\ell}}= & -Y_{\bar{\ell}} Y_{\gamma}\left(g+\log \left|\rho_{n+1}\right|^{2}\right)+\left(Y_{\alpha}\left(h^{\alpha}\right)+h^{\alpha} Y_{\alpha}(g)-2 h^{\alpha} h_{\alpha}\right. \\
& \left.+\frac{\rho_{n+1} \overline{n+1}}{\left|\rho_{n+1}\right|^{2}}-\frac{\rho_{n+1 n+1}}{\rho_{n+1}^{2}}\right) h_{\gamma \bar{\ell}} \\
= & -Y_{\bar{\ell}} Y_{\gamma}(\log J(\rho))+\left(Y_{\alpha}\left(h^{\alpha}\right)+h^{\alpha} Y_{\alpha} g(z)-2 h^{\alpha} h_{\alpha}\right. \\
& \left.+\frac{\rho_{n+1} \overline{n+1}}{\left|\rho_{n+1}\right|^{2}}-\frac{\rho_{n+1 n+1}}{\rho_{n+1}^{2}}\right) h_{\gamma \bar{\ell}},
\end{aligned}
$$

where $\alpha$ is summing from 1 to $n$.

The main result for this section is the following proof of Theorem 1.1. 
The proof of Theorem 1.1. We first assume that $H(\rho)$ is positive definite on $M$. Since

$$
\sum_{\alpha=1}^{n} \sum_{k=1}^{n+1} \rho^{\alpha \bar{k}} Y_{\alpha}\left(\rho_{\bar{k}}\right)=\sum_{\alpha=1}^{n} \sum_{k=1}^{n+1} \rho^{\alpha \bar{k}}\left(\rho_{\alpha \bar{k}}-\frac{\rho_{\alpha}}{\rho_{n+1}} \rho_{n+1 \bar{k}}\right)=n
$$

and

$$
Y_{\gamma}\left(\rho^{\alpha \bar{k}}\right)=-\sum_{p, q=1}^{n+1} \rho^{\alpha \bar{q}} \rho^{p \bar{k}} Y_{\gamma}\left(\rho_{p \bar{q}}\right)
$$

we have, by $h^{\alpha}=\rho^{\alpha} /|\partial \rho|_{\rho}^{2}$,

$$
\begin{aligned}
|\partial \rho|_{\rho}^{2} \sum_{\alpha=1}^{n} Y_{\alpha}\left(h^{\alpha}\right) & =\rho^{\alpha \bar{k}} Y_{\alpha}\left(\rho_{\bar{k}}\right)+\rho_{\bar{k}} Y_{\alpha}\left(\rho^{\alpha \bar{k}}\right)-h^{\alpha} Y_{\alpha}\left(|\partial \rho|_{\rho}^{2}\right) \\
& =n-\rho_{\bar{k}} \rho^{\alpha \bar{q}} \rho^{p \bar{k}} Y_{\alpha}\left(\rho_{p \bar{q}}\right)-h^{\alpha} Y_{\alpha}\left(|\partial \rho|_{\rho}^{2}\right) \\
& =n-|\partial \rho|_{\rho}^{2} \rho^{\alpha \bar{q}} h^{p} Y_{\alpha}\left(\rho_{p \bar{q}}\right)-h^{\alpha} Y_{\alpha}\left(|\partial \rho|_{\rho}^{2}\right)
\end{aligned}
$$

Since $J(\rho)=\operatorname{det} H(\rho)\left(|\partial \rho|_{\rho}^{2}-\rho\right)$, we have

$$
\begin{aligned}
\rho^{\alpha \bar{q}} h^{p} Y_{\alpha}\left(\rho_{p \bar{q}}\right) & =\rho^{\alpha \bar{q}} h^{p} \rho_{\alpha p \bar{q}}-\rho^{\alpha \bar{q}} h^{p} \frac{\rho_{\alpha}}{\rho_{n+1}} \rho_{n+1 p \bar{q}} \\
& =h^{p} \partial_{p} \log \operatorname{det} H(\rho)-\rho^{n+1 \bar{q}} h^{p} \rho_{n+1 p \bar{q}}-\frac{\rho^{\alpha \bar{q}} \rho_{\alpha}}{\rho_{n+1}} h^{p} \rho_{n+1 p \bar{q}} \\
& =h^{p} \partial_{p} \log \operatorname{det} H(\rho)-h^{p} \frac{\rho_{n+1 p \bar{q}}}{\rho_{n+1}}\left(\rho^{n+1 \bar{q}} \rho_{n+1}+\rho^{\alpha \bar{q}} \rho_{\alpha}\right) \\
& =h^{p} \partial_{p} \log \operatorname{det} H(\rho)-h^{p} \frac{\rho_{n+1 p \bar{q}}}{\rho_{n+1}} \rho^{\bar{q}} \\
& =h^{p} \partial_{p} \log J(\rho)-h^{p} \partial_{p} \log \left(|\partial \rho|_{\rho}^{2}-\rho\right)-h^{p} \frac{\rho_{n+1 p \bar{q}}}{\rho_{n+1}} \rho^{\bar{q}},
\end{aligned}
$$

where we sum $p$ and $q$ from 1 to $n+1$ and $\alpha$ from 1 to $n$. Since $\sum_{k=1}^{n+1} \rho_{k} h^{k}=$ $1, Y_{\alpha} \log |\partial \rho|_{\rho}^{2}=Y_{\alpha} \log \left(|\partial \rho|_{\rho}^{2}-\rho\right)$ and

$$
\frac{1}{\rho_{n+1}} \partial_{n+1} \log \left(|\partial \rho|_{\rho}^{2}-\rho\right)=\frac{1}{\rho_{n+1}} \frac{\partial}{\partial z_{n+1}} \log |\partial \rho|_{\rho}^{2}-\frac{1}{|\partial \rho|_{\rho}^{2}} \quad \text { on } M_{1}
$$


we have

$$
\begin{aligned}
\sum_{\alpha=1}^{n} Y_{\alpha}\left(h^{\alpha}\right)= & \frac{n}{|\partial \rho|_{\rho}^{2}}-h^{p} \partial_{p} \log J(\rho)+h^{p} \partial_{p} \log \left(|\partial \rho|_{\rho}^{2}-\rho\right)+\frac{h^{p} \rho^{\bar{q}} \rho_{n+1 p \bar{q}}}{\rho_{n+1}} \\
& -h^{\alpha} Y_{\alpha}\left(\log |\partial \rho|_{\rho}^{2}\right) \\
= & \frac{n}{|\partial \rho|_{\rho}^{2}}-h^{p} \partial_{p} \log J(\rho)+h^{n+1} \partial_{n+1} \log \left(|\partial \rho|_{\rho}^{2}-\rho\right)+\frac{h^{p} \rho^{\bar{q}} \rho_{n+1 p \bar{q}}}{\rho_{n+1}} \\
& +\frac{h^{\alpha} \rho_{\alpha}}{\rho_{n+1}} \partial_{n+1} \log \left(|\partial \rho|_{\rho}^{2}-\rho\right) \\
= & \frac{n}{|\partial \rho|_{\rho}^{2}}-h^{p} \partial_{p} \log J(\rho)+\frac{\sum_{p=1}^{n+1} \rho_{p} h^{p}}{\rho_{n+1}} \partial_{n+1} \log \left(|\partial \rho|_{\rho}^{2}\right)-\frac{1}{|\partial \rho|_{\rho}^{2}} \\
& +\frac{\partial_{n+1}\left(\rho^{p} \rho^{\bar{q}} \rho_{p \bar{q}}\right)}{|\partial \rho|_{\rho}^{2} \rho_{n+1}}-\frac{\partial_{n+1}\left(\rho^{p}\right) \rho^{\bar{q}} \rho_{p \bar{q}}}{|\partial \rho|_{\rho}^{2} \rho_{n+1}}-\frac{\rho^{p} \partial_{n+1}\left(\rho^{\bar{q}}\right) \rho_{p \bar{q}}}{|\partial \rho|_{\rho}^{2} \rho_{n+1}} \\
= & \frac{n-1}{|\partial \rho|_{\rho}^{2}}-h^{p} \partial_{p} \log J(\rho)+\frac{2}{\rho_{n+1}} \partial_{n+1} \log \left(|\partial \rho|_{\rho}^{2}\right)-\frac{\partial_{n+1}\left(\rho^{p}\right) \rho_{p}}{|\partial \rho|_{\rho}^{2} \rho_{n+1}} \\
& -\frac{\rho_{\bar{q}} \partial_{n+1}\left(\rho^{\bar{q}}\right)}{|\partial \rho|_{\rho}^{2} \rho_{n+1}} \\
= & \frac{n-1}{|\partial \rho|_{\rho}^{2}}-h^{p} \partial_{p} \log J(\rho)+\frac{2}{\rho_{n+1}} \partial_{n+1} \log \left(|\partial \rho|_{\rho}^{2}\right) \\
& -\frac{\partial_{n+1}\left(\rho^{p} \rho_{p}\right)-\rho^{p} \rho_{n+1 p}}{|\partial \rho|_{\rho}^{2} \rho_{n+1}} \frac{\partial_{n+1}\left(\rho_{\bar{q}} \rho^{\bar{q}}\right)-\rho^{\bar{q}} \rho_{n+1 \bar{q}}}{|\partial \rho|_{\rho}^{2} \rho_{n+1}} \\
= & \frac{n-1}{|\partial \rho|_{\rho}^{2}}-h^{p} \partial_{p} \log J(\rho)+\frac{2}{\rho_{n+1}} \partial_{n+1} \log \left(|\partial \rho|_{\rho}^{2}\right)-2 \frac{\partial_{n+1} \log |\partial \rho|_{\rho}^{2}}{\rho_{n+1}} \\
& +\frac{\rho^{p} \rho_{n+1 p}+\rho^{\bar{q}} \rho_{n+1 \bar{q}}}{|\partial \rho|_{\rho}^{2} \rho_{n+1}} \\
= & \frac{n-1}{|\partial \rho|_{\rho}^{2}}-h^{p} \partial_{p} \log J(\rho)+\frac{h^{p} \partial_{n+1}\left(\rho_{p}\right)+h^{\bar{q}} \partial_{n+1}\left(\rho_{\bar{q}}\right)}{\rho_{n+1}} \\
& \\
&
\end{aligned}
$$

Since

$$
\begin{aligned}
h^{\alpha} Y_{\alpha} g= & h^{\alpha} Y_{\alpha}\left(-\log \left|\rho_{n+1}\right|^{2}+\log J(\rho)\right) \\
= & -h^{\alpha} \frac{\rho_{\alpha n+1}}{\rho_{n+1}}-h^{\alpha} \frac{\rho_{\alpha \overline{n+1}}}{\rho_{\overline{n+1}}}+\frac{h^{\alpha} \rho_{\alpha}}{\rho_{n+1}}\left(\frac{\rho_{n+1 n+1}}{\rho_{n+1}}+\frac{\rho_{n+1 \overline{n+1}}}{\rho_{\overline{n+1}}}\right) \\
& +h^{\alpha} Y_{\alpha} \log J(\rho)
\end{aligned}
$$


and

$$
-h^{p} \partial_{p} \log J(\rho)+\sum_{\alpha=1}^{n} h^{\alpha} Y_{\alpha} \log J(\rho)=-\frac{1}{\rho_{n+1}} \partial_{n+1} \log J(\rho)
$$

we have

$$
\begin{aligned}
& \sum_{\alpha=1}^{n} Y_{\alpha}\left(h^{\alpha}\right)+h^{\alpha} Y_{\alpha} g(z)+\frac{\rho_{n+1 \overline{n+1}}}{\left|\rho_{n+1}\right|^{2}}-\frac{\rho_{n+1 n+1}}{\rho_{n+1}^{2}} \\
&=\frac{n-1}{|\partial \rho|_{\rho}^{2}}-\frac{1}{\rho_{n+1}} \partial_{n+1} \log J(\rho)+\frac{h^{p} \partial_{n+1}\left(\rho_{p}\right)+h^{\bar{q}} \partial_{n+1}\left(\rho_{\bar{q}}\right)}{\rho_{n+1}}-h^{\alpha} \frac{\rho_{\alpha n+1}}{\rho_{n+1}} \\
&-h^{\alpha} \frac{\rho_{\alpha \overline{n+1}}}{\rho_{\overline{n+1}}}+\frac{h^{\alpha} \rho_{\alpha}}{\rho_{n+1}}\left(\frac{\rho_{n+1 n+1}}{\rho_{n+1}}+\frac{\rho_{n+1} \overline{n+1}}{\rho_{\overline{n+1}}}\right)+\frac{\rho_{n+1 \overline{n+1}}}{\left|\rho_{n+1}\right|^{2}}-\frac{\rho_{n+1 n+1}}{\rho_{n+1}^{2}} \\
&= \frac{n-1}{|\partial|_{\rho}^{2}}-\frac{1}{\rho_{n+1}} \partial_{n+1} \log J(\rho)+\frac{h^{n+1} \rho_{n+1 n+1}}{\rho_{n+1}}+\frac{h^{\overline{n+1}} \rho_{n+1} \overline{n+1}}{\rho_{n+1}} \\
&+\left(-\frac{h^{\alpha}}{\rho_{\overline{n+1}}} \rho_{\alpha \overline{n+1}}+\frac{h^{\bar{\alpha}}}{\rho_{n+1}} \rho_{n+1 \bar{\alpha}}\right)+\frac{h^{\alpha} \rho_{\alpha}}{\rho_{n+1}}\left(\frac{\rho_{n+1 n+1}}{\rho_{n+1}}+\frac{\rho_{n+1} \overline{n+1}}{\rho_{\overline{n+1}}}\right) \\
&+\frac{\rho_{n+1} \overline{n+1}}{\left|\rho_{n+1}\right|^{2}}-\frac{\rho_{n+1 n+1}}{\rho_{n+1}^{2}} \\
&= \frac{n-1}{|\partial \rho|_{\rho}^{2}}-\frac{1}{\rho_{n+1}} \partial_{n+1} \log J(\rho)+\frac{\rho_{n+1 n+1}}{\rho_{n+1}^{2}}+\frac{\rho_{\overline{n+1}} h^{\overline{n+1}} \rho_{n+1} \overline{\mid \rho_{n+1}}}{\left|\rho_{n+1}\right|^{2}} \\
&+\left(-\frac{h^{\alpha}}{\rho_{\overline{n+1}}} \rho_{\alpha \overline{n+1}}+\frac{h^{\bar{\alpha}}}{\rho_{n+1}} \rho_{n+1 \bar{\alpha}}\right)+\frac{h^{\alpha} \rho_{\alpha}}{\rho_{n+1}}\left(\frac{\rho_{n+1} \overline{n+1}}{\rho_{\overline{n+1}}}\right)+\frac{\rho_{n+1} \overline{n+1}}{\left|\rho_{n+1}\right|^{2}} \\
&-\frac{\rho_{n+1 n+1}}{\rho_{n+1}^{2}} \\
&= \frac{n-1}{|\partial \rho|_{\rho}^{2}}-\frac{1}{\rho_{n+1}} \partial_{n+1} \log J(\rho)+2 \frac{\rho_{n+1} \overline{n+1}}{\left|\rho_{n+1}\right|^{2}}+E(\rho),
\end{aligned}
$$

where

$$
E(\rho):=\sum_{\alpha=1}^{n}\left(-\frac{h^{\alpha}}{\rho_{\overline{n+1}}} \rho_{\alpha \overline{n+1}}+\frac{h^{\bar{\alpha}}}{\rho_{n+1}} \rho_{n+1 \bar{\alpha}}\right)+\left(h^{\alpha} \rho_{\alpha}-h^{\bar{\alpha}} \rho_{\bar{\alpha}}\right) \frac{\rho_{n+1} \overline{n+1}}{\left|\rho_{n+1}\right|^{2}}
$$

is a pure imaginary number. 
For $1 \leq p, q \leq n+1$, we let

$$
B_{p \bar{q}}:=\sum_{\alpha, \beta=1}^{n} h_{\alpha \bar{\beta}} \rho^{\alpha \bar{q}} \rho^{p \bar{\beta}} .
$$

Then,

$$
\begin{aligned}
B_{p \bar{q}}= & \sum_{\alpha, \beta=1}^{n}\left(\rho_{\alpha \bar{\beta}}-\frac{\rho_{\alpha} \rho_{n+1} \bar{\beta}}{\rho_{n+1}}-\frac{\rho_{\alpha \overline{n+1}} \rho_{\bar{\beta}}}{\rho_{\overline{n+1}}}+\frac{\rho_{n+1} \overline{n+1}}{\left|\rho_{n+1}\right|^{2}} \rho_{\alpha} \rho_{\bar{\beta}}\right) \rho^{\alpha \bar{q}} \rho^{p \bar{\beta}} \\
= & \sum_{\beta=1}^{n} \rho^{p \bar{\beta}}\left(\delta_{q \beta}-\rho_{n+1 \bar{\beta}} \rho^{n+1 \bar{q}}\right)-\sum_{\alpha=1}^{n} \frac{\rho_{\alpha}}{\rho_{n+1}}\left(\delta_{p n+1}-\rho_{n+1 \overline{n+1}} \rho^{p \overline{n+1}}\right) \rho^{\alpha \bar{q}} \\
& -\sum_{\beta=1}^{n} \frac{\rho_{\bar{\beta}}}{\rho_{\overline{n+1}}}\left(\delta_{n+1 q}-\rho^{n+1 \bar{q}} \rho_{n+1 \overline{n+1}}\right) \rho^{p \bar{\beta}}+\frac{\rho_{n+1} \overline{n+1}}{\left|\rho_{n+1}\right|^{2}} \rho_{\alpha} \rho_{\bar{\beta}} \rho^{\alpha \bar{q}} \rho^{p \bar{\beta}}
\end{aligned}
$$

and

$$
\begin{aligned}
\sum_{\alpha=1}^{n} h^{\alpha} h_{\alpha}|\partial \rho|_{\rho}^{4}= & \sum_{\alpha, \beta=1}^{n} h_{\alpha \bar{\beta}} h^{\alpha} h^{\bar{\beta}}|\partial \rho|_{\rho}^{4} \\
= & \sum_{p, q=1}^{n+1} B_{p \bar{q}} \rho_{p} \rho_{\bar{q}} \\
= & \sum_{p, q=1}^{n+1} \rho_{p} \rho_{\bar{q}} \sum_{\beta=1}^{n} \rho^{p \bar{\beta}}\left(\delta_{q \beta}-\rho_{n+1 \bar{\beta}} \rho^{n+1 \bar{q}}\right) \\
& -\sum_{p, q=1}^{n+1} \rho_{p} \rho_{\bar{q}} \sum_{\alpha=1}^{n} \frac{\rho_{\alpha}}{\rho_{n+1}}\left(\delta_{p n+1}-\rho_{n+1 \overline{n+1}} \rho^{p \overline{n+1}}\right) \rho^{\alpha \bar{q}} \\
& -\sum_{p, q=1}^{n+1} \rho_{p} \rho_{\bar{q}} \sum_{\beta=1}^{n} \frac{\rho_{\bar{\beta}}}{\rho_{\overline{n+1}}}\left(\delta_{n+1 q}-\rho^{n+1 \bar{q}} \rho_{n+1} \overline{n+1}\right) \rho^{p \bar{\beta}} \\
& +\sum_{p, q=1}^{n+1} \rho_{p} \rho_{\bar{q}} \frac{\rho_{n+1}}{\left|\rho_{n+1}\right|^{2}} \rho_{\alpha} \rho_{\bar{\beta}} \rho^{\alpha \bar{q}} \rho^{p \bar{\beta}} \\
= & \sum_{\beta=1}^{n} \rho^{\bar{\beta}} \rho_{\bar{\beta}}-\rho^{n+1} \rho_{n+1}+\rho_{n+1} \overline{n+1} \rho^{n+1} \rho^{\overline{n+1}} \\
& -\sum_{\alpha=1}^{n} \rho^{\alpha} \rho_{\alpha}+\sum_{\alpha=1}^{n} \rho^{\alpha} \rho_{\alpha} \frac{\rho^{n+1}}{\rho_{n+1}} \rho_{n+1} \overline{n+1}-\sum_{\beta=1}^{n} \rho^{\bar{\beta}} \rho_{\bar{\beta}} \\
& +\sum_{\beta=1}^{n} \rho^{\bar{\beta}} \rho_{\bar{\beta}} \frac{\rho^{n+1}}{\rho_{\overline{n+1}}} \rho_{n+1} \overline{n+1}+\sum_{\alpha, \beta=1}^{n} \rho^{\alpha} \rho_{\alpha} \rho^{\bar{\beta}} \rho_{\bar{\beta}} \frac{\rho_{n+1} \overline{n+1}}{\left|\rho_{n+1}\right|^{2}}
\end{aligned}
$$




$$
\begin{aligned}
= & -|\partial \rho|_{\rho}^{2}+\frac{\rho_{n+1} \overline{n+1}}{\left|\rho_{n+1}\right|^{2}} \rho_{n+1} \rho^{n+1} \rho_{\overline{n+1}} \rho^{\overline{n+1}} \\
& +\left[\sum_{\alpha=1}^{n} \rho^{\alpha} \rho_{\alpha} \rho^{\overline{n+1}} \rho_{n+1}+\sum_{\beta=1}^{n} \rho^{\bar{\beta}} \rho_{\bar{\beta}} \rho^{n+1} \rho_{n+1}\right] \frac{\rho_{n+1 \overline{n+1}}}{\left|\rho_{n+1}\right|^{2}} \\
& +\sum_{\alpha, \beta=1}^{n} \rho^{\alpha} \rho_{\alpha} \rho^{\bar{\beta}} \rho_{\bar{\beta}} \frac{\rho_{n+1} \overline{n+1}}{\left|\rho_{n+1}\right|^{2}} \\
= & -|\partial \rho|_{\rho}^{2}+|\partial \rho|^{4} \frac{\rho_{n+1} \overline{n+1}}{\left|\rho_{n+1}\right|^{2}} .
\end{aligned}
$$

Therefore,

$$
\begin{aligned}
Y^{\alpha} & \left(h_{\alpha}\right)+h^{\alpha} Y_{\alpha} g(z)-2 h^{\alpha} h_{\alpha}+\frac{\rho_{n+1} \overline{n+1}}{\left|\rho_{n+1}\right|^{2}}-\frac{\rho_{n+1 n+1}}{\rho_{n+1}^{2}} \\
\quad= & \frac{n-1}{|\partial \rho|_{\rho}^{2}}-\frac{1}{\rho_{n+1}} \partial_{n+1} \log J(\rho)+2 \frac{\rho_{n+1} \overline{n+1}}{\left|\rho_{n+1}\right|^{2}}+\frac{2}{|\partial \rho|_{\rho}^{2}}-2 \frac{\rho_{n+1} \overline{n+1}}{\left|\rho_{n+1}\right|^{2}}+E(\rho) \\
\quad= & \frac{n+1}{|\partial \rho|_{\rho}^{2}}-\frac{1}{\rho_{n+1}} \partial_{n+1} \log J(\rho)+E(\rho) .
\end{aligned}
$$

Notice that $R_{\alpha \bar{\beta}}=\overline{R_{\beta \bar{\alpha}}}, h_{\alpha \bar{\beta}}=\overline{h_{\beta \bar{\alpha}}}$ and $\overline{E(\rho)}=-E(\rho)$ (by $(2.37), E(\rho)$ is pure imaginary). By (2.32), we have

$$
\begin{aligned}
R_{\gamma \bar{\ell}}= & \frac{1}{2}\left(R_{\gamma \bar{\ell}}+\overline{R_{\ell \bar{\gamma}}}\right) \\
= & -\frac{1}{2}\left(Y_{\bar{\ell}} Y_{\gamma}+Y_{\gamma} Y_{\bar{\ell}}\right) \log J(\rho) \\
& +\left(\frac{n+1}{|\partial \rho|_{\rho}^{2}}-\frac{1}{2}\left(\frac{1}{\rho_{n+1}} \partial_{n+1}+\frac{1}{\rho_{\overline{n+1}}} \partial_{\overline{n+1}}\right) \log J(\rho)\right) h_{\gamma \bar{\ell}}
\end{aligned}
$$

Since

$$
\begin{aligned}
Y_{\bar{\ell}} Y_{\gamma}= & \frac{\partial^{2}}{\partial \bar{z}^{\ell} \partial z^{\gamma}}-\frac{\partial}{\partial \bar{z}^{\ell}}\left(\frac{\rho_{\gamma}}{\rho_{n+1}} \partial_{n+1}\right)-\frac{\rho_{\bar{\ell}}}{\rho_{\overline{n+1}}} \frac{\partial^{2}}{\partial \bar{z}^{n+1} \partial z^{\gamma}} \\
& +\frac{\rho_{\bar{\ell}}}{\rho_{\overline{n+1}}} \partial_{\overline{n+1}}\left(\frac{\rho_{\gamma}}{\rho_{n+1}} \partial_{n+1}\right)
\end{aligned}
$$




$$
\begin{aligned}
= & \frac{\partial^{2}}{\partial \bar{z}^{\ell} \partial z^{\gamma}}+\left[-\frac{\rho_{\gamma \bar{\ell}}}{\rho_{n+1}}+\frac{\rho_{\gamma}}{\rho_{n+1}^{2}} \rho_{n+1 \bar{\ell}}\right] \partial_{n+1}-\frac{\rho_{\gamma}}{\rho_{n+1}} \frac{\partial^{2}}{\partial z^{n+1} \partial \bar{z}^{\ell}} \\
& -\frac{\rho_{\bar{\ell}}}{\rho_{\overline{n+1}}} \frac{\partial^{2}}{\partial \bar{z}^{n+1} \partial z^{\gamma}}+\frac{\rho_{\bar{\ell}}}{\rho_{\overline{n+1}}} \frac{\rho_{\gamma \overline{n+1}}}{\rho_{n+1}} \partial_{n+1}-\frac{\rho_{\bar{\ell}}}{\rho_{\overline{n+1}}} \frac{\rho_{\gamma} \rho_{n+1} \overline{n+1}}{\rho_{n+1}^{2}} \partial_{n+1} \\
& +\frac{\rho_{\bar{\ell}}}{\rho_{\overline{n+1}}} \frac{\rho_{\gamma}}{\rho_{n+1}} \frac{\partial^{2}}{\partial z^{n+1} \partial \bar{z}^{n+1}} \\
= & -\frac{h_{\gamma \bar{\ell}}}{\rho_{n+1}} \partial_{n+1}+\frac{\partial^{2}}{\partial \bar{z}^{\ell} \partial z^{\gamma}}-\frac{\rho_{\gamma}}{\rho_{n+1}} \frac{\partial^{2}}{\partial z^{n+1} \partial \bar{z}^{\ell}}-\frac{\rho_{\bar{\ell}}}{\rho_{\overline{n+1}}} \frac{\partial^{2}}{\partial \bar{z}^{n+1} \partial z^{\gamma}} \\
& +\frac{\rho_{\gamma} \rho_{\bar{\ell}}}{\left|\rho_{n+1}\right|^{2}} \frac{\partial^{2}}{\partial z^{n+1} \partial \bar{z}^{n+1}}
\end{aligned}
$$

we have

$$
Y_{\bar{\ell}} Y_{\gamma}=-\frac{h_{\gamma \bar{\ell}}}{\rho_{n+1}} \partial_{n+1}+\mathcal{D}_{\gamma \bar{\ell}}^{\rho}
$$

where

$$
\mathcal{D}_{\gamma \bar{\ell}}^{\rho}=\frac{\partial^{2}}{\partial \bar{z}^{\ell} \partial z^{\gamma}}-\frac{\rho_{\gamma}}{\rho_{n+1}} \frac{\partial^{2}}{\partial z^{n+1} \partial \bar{z}^{\ell}}-\frac{\rho_{\bar{\ell}}}{\rho_{\overline{n+1}}} \frac{\partial^{2}}{\partial \bar{z}^{n+1} \partial z^{\gamma}}+\frac{\rho_{\gamma} \rho_{\bar{\ell}}}{\left|\rho_{n+1}\right|^{2}} \frac{\partial^{2}}{\partial z^{n+1} \partial \bar{z}^{n+1}} .
$$

Then,

$$
h_{\alpha \bar{\beta}}=\mathcal{D}_{\alpha \bar{\beta}}^{\rho}(\rho), \quad \overline{\mathcal{D}_{\beta \bar{\alpha}}^{\rho}}=\mathcal{D}_{\alpha \bar{\beta}}^{\rho}
$$

and

$$
Y_{\bar{\beta}} Y_{\alpha}+Y_{\alpha} Y_{\bar{\beta}}=-\frac{h_{\alpha \bar{\beta}}}{\rho_{n+1}} \partial_{n+1}-\frac{h_{\alpha \bar{\beta}}}{\rho_{\overline{n+1}}} \partial_{\overline{n+1}}+2 \mathcal{D}_{\alpha \bar{\beta}}^{\rho} .
$$

By (2.38) and (2.42), we have

$$
R_{\alpha \bar{\beta}}=-\mathcal{D}_{\alpha \bar{\beta}}^{\rho} \log J(\rho)+(n+1) \frac{\operatorname{det} H(\rho)}{J(\rho)} \mathcal{D}_{\alpha \bar{\beta}}^{\rho}(\rho)
$$

since $J(\rho)=|\partial \rho|_{\rho}^{2}(\operatorname{det} H(\rho)-\rho)$. 
In order to prove (1.6), for any $z_{0} \in M_{1}$, we let

$$
w=\sum_{j=1}^{n+1} w^{j} \frac{\partial}{\partial z^{j}}, \quad v=\sum_{j=1}^{n+1} v^{j} \frac{\partial}{\partial z^{j}} \in\left(T_{1,0}\right)_{z_{0}}(M) .
$$

Then,

$$
\sum_{j=1}^{n+1} w^{j} \rho_{j}\left(z_{0}\right)=\sum_{j=1}^{n+1} v^{j} \rho_{j}\left(z_{0}\right)=0 .
$$

It is easy to see that

$$
w=\sum_{j=1}^{n} w^{j} Y_{j}, \quad v=\sum_{j=1}^{n} v^{j} Y_{j}
$$

Thus,

$\operatorname{Ric}(w, \bar{v})\left(z_{0}\right)=\sum_{\alpha, \beta=1}^{n} R_{\alpha \bar{\beta}} w^{\alpha} \bar{v}^{\beta}$

$$
=-\sum_{\alpha, \beta=1}^{n} w^{\alpha} \bar{v}^{\beta} \mathcal{D}_{\alpha \bar{\beta}}^{\rho} \log J(\rho)+(n+1) \frac{\operatorname{det} H(\rho)}{J(\rho)} \sum_{\alpha, \beta=1}^{n} h_{\alpha \bar{\beta}} w^{\alpha} \bar{v}^{\beta} .
$$

Applying (2.45), we have

$$
\begin{aligned}
\sum_{\alpha, \beta=1}^{n} w^{\alpha} \bar{v}^{\beta} \mathcal{D}_{\alpha \bar{\beta}}^{\rho}= & \sum_{\alpha, \beta=1}^{n} w^{\alpha} \bar{v}^{\beta} \frac{\partial^{2}}{\partial z^{\alpha} \partial \bar{z}^{\beta}}-\sum_{\alpha, \beta=1}^{n} \frac{\rho_{\bar{\beta}}}{\rho \overline{n+1}} w^{\alpha} \bar{v}^{\beta} \frac{\partial^{2}}{\partial z^{\alpha} \partial \bar{z}^{n+1}} \\
& -\sum_{\alpha, \beta=1}^{n} \frac{\rho_{\alpha}}{\rho_{n+1}} w^{\alpha} \bar{v}^{\beta} \frac{\partial^{2}}{\partial z^{n+1} \partial \bar{z}^{\beta}} \\
& +\sum_{\alpha, \beta=1}^{n} \frac{\rho_{\alpha} \rho_{\bar{\beta}}}{\left|\rho_{n+1}\right|^{2}} w^{\alpha} \bar{v}^{\beta} \frac{\partial^{2}}{\partial z^{n+1} \partial \bar{z}^{n+1}} \\
= & \sum_{\alpha, \beta=1}^{n} w^{\alpha} \bar{v}^{\beta} \frac{\partial^{2}}{\partial z^{\alpha} \partial \bar{z}^{\beta}}+\sum_{\alpha=1}^{n} w^{\alpha} \bar{v}^{n+1} \frac{\partial^{2}}{\partial z^{\alpha} \partial \bar{z}^{n+1}} \\
& +\sum_{\beta=1}^{n} w^{n+1} \bar{v}^{\beta} \frac{\partial^{2}}{\partial z^{n+1} \partial \bar{z}^{\beta}}+w^{n+1} \bar{v}^{n+1} \frac{\partial^{2}}{\partial z^{n+1} \partial \bar{z}^{n+1}} \\
= & \sum_{k, j=1}^{n+1} w^{k} \bar{v}^{j} \frac{\partial^{2}}{\partial z^{k} \partial \bar{z}^{j}} .
\end{aligned}
$$


Similarly,

$$
\sum_{\alpha, \beta=1}^{n} h_{\alpha \bar{\beta}} w^{\alpha} \bar{v}^{\beta}=\sum_{k, j=1}^{n+1} \frac{\partial^{2} \rho\left(z_{0}\right)}{\partial z^{k} \partial \bar{z}^{j}} w^{k} \bar{v}^{j} .
$$

Combining (2.46), (2.47) and (2.48), we have proved formula (1.6).

In particular, if $\log J(\rho)$ is pluriharmonic near $\partial D$, then $\mathcal{D}_{\alpha \bar{\beta}}^{\rho} \log J(\rho)=0$, and

$$
R_{\gamma \bar{\ell}}\left(z_{0}\right)=(n+1) \frac{\operatorname{det} H(\rho)}{J(\rho)} h_{\gamma \bar{\ell}}\left(z_{0}\right) .
$$

This with (2.46) and (2.48) gives the formula (1.7). Therefore, Theorem 1.1 is proved when $H(\rho)$ is positive definite at $z_{0} \in M_{1}$.

Next, we consider the case when $H(\rho)$ may not be positive definite. Instead of $\rho$, we use $r(z)=\rho+c \rho^{2}$ with $c>0$ being chosen so that $H(r)$ is positive definite on $M$. Moreover, the contact form $\theta$ remains the same if we use $r$ replacing $\rho$. Then (1.6) holds by replacing $\rho$ by $r$. We will show the right side is the same for $\rho$ and for $r$. We know $J(r)=J(\rho)$ on $M$. However, $J(r)$ will be different in $D$. Notice that

$$
J(r)=\left|r_{n+1}\right|^{2} \operatorname{det}\left(h_{\alpha \bar{\beta}}(r)\right)-r(z) \operatorname{det}(H(r))
$$

and

$$
\begin{aligned}
h_{\alpha \bar{\beta}}(r)= & r_{\alpha \bar{\beta}}-\frac{r_{\alpha \overline{n+1}}}{r_{\overline{n+1}}} r_{\bar{\beta}}-\frac{r_{n+1 \bar{\beta}}}{r_{n+1}} r_{\alpha}+r_{n+1 \overline{n+1}} \frac{r_{\alpha} r_{\bar{\beta}}}{\left|r_{n+1}\right|^{2}} \\
= & (1+2 c \rho) \rho_{\alpha \bar{\beta}}+2 c \rho_{\alpha} \rho_{\bar{\beta}}-\frac{(1+2 c \rho) \rho_{\alpha \overline{n+1}}+2 c \rho_{\alpha} \rho_{\overline{n+1}}}{(1+2 c \rho) \rho_{\overline{n+1}}}(1+2 c \rho) \rho_{\bar{\beta}} \\
& -\frac{(1+2 c \rho) \rho_{n+1 \bar{\beta}}+2 c \rho_{n+1} \rho_{\bar{\beta}}}{(1+2 c \rho) \rho_{n+1}}(1+2 c \rho) \rho_{\alpha} \\
& +\left((1+2 c \rho) \rho_{n+1 \overline{n+1}}+2 c\left|\rho_{n+1}\right|^{2}\right) \frac{(1+2 c \rho)^{2} \rho_{\alpha} \rho_{\bar{\beta}}}{(1+2 c \rho)^{2}\left|\rho_{n+1}\right|^{2}} \\
= & (1+2 c \rho) \rho_{\alpha \bar{\beta}}+2 c \rho_{\alpha} \rho_{\bar{\beta}}-\frac{(1+2 c \rho) \rho_{\alpha \overline{n+1}}+2 c \rho_{\alpha} \rho_{\overline{n+1}}}{\rho_{\overline{n+1}}} \rho_{\bar{\beta}} \\
& -\frac{(1+2 c \rho) \rho_{n+1 \bar{\beta}}+2 c \rho_{n+1} \rho_{\bar{\beta}}}{\rho_{n+1}} \rho_{\alpha} \\
& +\left((1+2 c \rho) \rho_{n+1 \overline{n+1}}+2 c\left|\rho_{n+1}\right|^{2}\right) \frac{\rho_{\alpha} \rho_{\bar{\beta}}}{\left|\rho_{n+1}\right|^{2}} \\
= & (1+2 c \rho) h_{\alpha \bar{\beta}}(\rho) .
\end{aligned}
$$


Thus,

$$
\begin{aligned}
J(r)= & (1+2 c \rho)^{n+2}\left|\rho_{n+1}\right|^{2} \operatorname{det}\left(h_{\alpha \bar{\beta}}(\rho)\right)-\rho(1+c \rho) \operatorname{det} H\left(\rho+c \rho^{2}\right) \\
= & (1+2 c \rho)^{n+2} J(\rho)+(1+2 c \rho)^{n+2} \rho(z) \operatorname{det}(H(\rho)) \\
& -\rho(1+c \rho) \operatorname{det} H\left(\rho+c \rho^{2}\right) \\
= & (1+2 c \rho)^{n+2} J(\rho)+\rho\left[\operatorname{det} H(\rho)-\operatorname{det} H\left(\rho+c \rho^{2}\right)\right]+O\left(\rho^{2}\right) \\
= & J(\rho)+\rho\left[2(n+2) c J(\rho)+\operatorname{det} H(\rho)-\operatorname{det} H\left(\rho+c \rho^{2}\right)\right]+O\left(\rho^{2}\right) .
\end{aligned}
$$

It is easy to see from the definition that on $M$ we have

$$
\mathcal{D}_{\alpha \bar{\beta}}^{r}=\mathcal{D}_{\alpha \bar{\beta}}^{\rho}
$$

and

$$
\begin{aligned}
\mathcal{D}_{\alpha \bar{\beta}}^{r} \log J(r)= & \mathcal{D}_{\alpha \bar{\beta}}^{\rho} \log J(\rho) \\
& +\left(2(n+2) c+\frac{\operatorname{det} H(\rho)-\operatorname{det} H\left(\rho+c \rho^{2}\right)}{J(\rho)}\right) \mathcal{D}_{\alpha \bar{\beta}}^{\rho}(\rho) \\
R_{\alpha \bar{\beta}}(r)\left(z_{0}\right)= & -\mathcal{D}_{\alpha \bar{\beta}}^{r} \log J(r)+(n+1) \frac{\operatorname{det} H(r)}{J(r)}(z) h_{\alpha \bar{\beta}} \\
= & -\mathcal{D}_{\alpha \bar{\beta}}^{\rho} \log J(\rho)-2(n+2) c h_{\alpha \bar{\beta}}+\frac{\operatorname{det} H(r)-\operatorname{det} H(\rho)}{J(\rho)\left(z_{0}\right)} h_{\alpha \bar{\beta}}(z) \\
& +(n+1) \frac{\operatorname{det} H(r)}{J(\rho)} h_{\alpha \bar{\beta}}(z) \\
= & -\mathcal{D}_{\alpha \bar{\beta}}^{\rho} \log J(\rho)+(n+1) \frac{\operatorname{det} H(\rho)}{J(\rho)} h_{\alpha \bar{\beta}}(z) \\
& +\frac{(n+2)}{J(\rho)}\left[\operatorname{det} H(r)-\operatorname{det} H(\rho)-2 c J(\rho)\left(z_{0}\right)\right] h_{\alpha \bar{\beta}}\left(z_{0}\right) \\
= & -\mathcal{D}_{\alpha \bar{\beta}}^{\rho} \log J(\rho)+(n+1) \frac{\operatorname{det} H(\rho)}{J(\rho)} h_{\alpha \bar{\beta}}\left(z_{0}\right),
\end{aligned}
$$

provided

$$
\phi(c)=\operatorname{det} H\left(\rho+c \rho^{2}\right)-\operatorname{det} H(\rho)-2 c J(\rho) \equiv 0 .
$$

Let $z_{0} \in M_{1}$. Then, we will prove that $(2.50)$ holds in the following two cases:

(a) If $\operatorname{det} H(\rho)\left(z_{0}\right) \neq 0$, then $\operatorname{det} H(r)\left(z_{0}\right)=\operatorname{det}(H(\rho)+2 c \partial \rho \otimes \bar{\partial} \rho)=\operatorname{det} H(\rho)\left(1+2 c|\partial \rho|_{\rho}^{2}\right)$, 
where $|\partial \rho|_{\rho}^{2}=\rho^{i \bar{j}} \rho_{i} \rho_{\bar{j}}\left(z_{0}\right)$ (it may not be positive). Thus,

$$
\phi^{\prime}(c)=\operatorname{det} H(\rho)\left(z_{0}\right) 2|\partial \rho|_{\rho}^{2}-2 J(\rho)=2(J(\rho)-J(\rho))=0 .
$$

Thus,

$$
\phi(c)=\phi(0)=0
$$

(b) If $\operatorname{det} H(\rho)\left(z_{0}\right)=0$, then $\operatorname{det} H\left(\rho+\epsilon \rho^{2}\right)\left(z_{0}\right) \neq 0$ for any $\epsilon>0$. Thus,

$$
\phi(c)=\operatorname{det} H\left(\rho+\epsilon \rho^{2}+(c-\epsilon) \rho^{2}\right)-2 c J(\rho)-\operatorname{det} H(\rho) .
$$

Thus,

$$
\phi^{\prime}(c)=2 J\left(\rho+\epsilon \rho^{2}\right)-2 J(\rho), \quad \text { for any small } \epsilon>0 .
$$

Let $\epsilon \rightarrow 0^{+}$, we have $\phi^{\prime}(c)=0$. Thus, $\phi(c)=\phi(0)=0$. The proof of the theorem is complete.

Furthermore, we will show that the pseudo-scalar curvatures are the same for those $\rho$ with $J(\rho)=1$ on $M$ and for $\rho^{0}$ with $J\left(\rho^{0}\right)=1$ in a neighborhood of $M$.

Corollary 2.3. Let $D$ be a strictly pseudo-convex domain in $\mathbb{C}^{n+1}$ with smooth boundary $M$. Let $\rho^{0}$ be a defining function of $D$ with $J\left(\rho^{0}\right) \equiv 1$ in a neighborhood $U$ of $M$, and let $\rho \in C^{3}(\bar{D})$ be any defining function for $D$ with $J(\rho)>0$ on $\bar{D} \cap U$ and $\theta=(\partial \rho-\bar{\partial} \rho) /(2 i)$. Then, for $v, w \in T_{1,0}(M)$, we have two conclusions:

(a) If $J(\rho)=1$ on $M$ or $J(\rho)=1+O\left(\rho^{0}\right)$, then

$$
\operatorname{Ric}(w, \bar{v})=(n+1) \operatorname{det} H\left(\rho^{0}\right) L_{\theta}(w, \bar{v}) .
$$

(b) If $\log J(\rho)$ is pluriharmonic near $M$, then

$$
\operatorname{Ric}(w, \bar{v})=(n+1) \frac{\operatorname{det} H(\rho)}{J(\rho)} L_{\theta}(w, \bar{v}) .
$$

Proof. We can write $\rho=a(z) \rho^{0}$ with $a \neq 0$ on $M$. Since $1=J(\rho)=J\left(a \rho^{0}\right)=$ $a^{n+2} J\left(\rho^{0}\right)$ on $M$, we have $a \equiv 1$ on $M$. Thus, we can write $a(z)=1+b \rho^{0}(z)$ and

$$
\rho(z)=\rho^{0}(z)+b(z) \rho^{0}(z)^{2} .
$$

It is not difficult to see that the last argument of the proof of the last theorem remains true when $c$ is a function (or since $\theta(\rho)=\theta\left(\rho^{0}\right)$ on $M$, they must 
have the same Ricci curvature). Therefore,

$$
R_{\alpha \bar{\beta}}(\rho)=(n+1) \operatorname{det} H\left(\rho^{0}\right) h_{\alpha \bar{\beta}} .
$$

This with (2.46) and (2.48) gives (2.51). Moreover, (2.52) follows directly from (1.6) which we have proved. Therefore, the proof of the corollary is complete.

\section{Proof of Theorem 1.2}

In order to prove Theorem 1.2, we recall a theorem proved by $\mathrm{Li}$ (see Theorem 1.1 in [23]). Let

$$
U(z)=-\log (-\rho(z)), \quad z \in D \subset \mathbb{C}^{n+1}
$$

be strictly plurisubharmonic in $D$. Let

$$
|\partial U|_{U}^{2}=\sum_{i, j=1}^{n+1} U^{i \bar{j}} U_{i} U_{\bar{j}}, \quad H(U)=\left[U_{i \bar{j}}\right]_{n \times n}, \quad\left[U^{i \bar{j}}\right]=\left(H(U)^{t}\right)^{-1} .
$$

Then,

$$
\begin{aligned}
\operatorname{det} H(\rho) & =\operatorname{det} H\left(-\mathrm{e}^{-U}\right) \\
& =\mathrm{e}^{-(n+1) U} \operatorname{det}\left(H(U)-(\bar{\partial} U)^{*}(\bar{\partial} U)\right) \\
& =\mathrm{e}^{-(n+1) U} \operatorname{det}(H(U))\left(1-|\partial U|_{U}^{2}\right)
\end{aligned}
$$

and

$$
J(\rho) \mathrm{e}^{(n+2) U}=-\operatorname{det}\left[\begin{array}{cc}
-1 & \bar{\partial} U \\
(\bar{\partial} U)^{*} & H(U)-(\bar{\partial} U)^{*}(\bar{\partial} U)
\end{array}\right]=\operatorname{det} H(U) .
$$

The following theorem is proved in [23]:

Theorem 3.1. Let $D$ be a bounded strictly pseudo-convex domain in $\mathbb{C}^{n+1}$ with smooth boundary. Let $\rho \in C^{3}(\bar{D})$ be a defining function for $D$ with $J(\rho)>0$ and $\log J(\rho)$ being pluriharmonic in D. Then the following conclusions hold:

(i) The function $\operatorname{det} H(\rho) / J(\rho)$ attains its minimum over $\bar{D}$ at some point in $\partial D$. 
(ii) If

$$
\frac{\operatorname{det} H(\rho)}{J(\rho)} \geq \frac{\operatorname{det} H(\rho)\left(z_{0}\right)}{J(\rho)\left(z_{0}\right)}, \quad \text { on } \partial D
$$

for some $z_{0} \in\{z \in D: \rho(z)=\min \{\rho(w): w \in \bar{D}\}\}$, then $D$ is biholomorphically equivalent to the unit ball in $\mathbb{C}^{n+1}$.

(iii) In addition to the condition (3.5), if we assume that $J(\rho) \equiv 1$ on $D$, then $D$ is biholomorphically equivalent to the unit ball in $\mathbb{C}^{n+1}$ with a constant Jacobian biholomorphic map.

We are ready to prove Theorem 1.2.

Proof. Let $\rho_{0}(z)$ be the plurisubharmonic solution of the Monge-Ampère equation (given in [4]):

$$
\operatorname{det} H\left(\rho_{0}\right)=J(\rho) \quad \text { in } D, \quad \rho_{0}=0 \text { on } \partial D \text {. }
$$

Since $\log J(\rho)$ is pluriharmonic, by formula (2.43) or (1.6), we have

$$
R_{\theta}=n(n+1) \frac{\operatorname{det} H(\rho)}{J(\rho)}, \quad \text { on } \partial D
$$

Combining this with assumption (1.9) $\left(\operatorname{Area}_{\theta}(\partial D)=\int_{\partial D} \theta \wedge(d \theta)^{n}\right)$, we have

$$
\frac{\operatorname{det} H(\rho)}{J(\rho)} \geq \frac{\int_{\partial D} \theta \wedge(d \theta)^{n}}{\int_{\partial D} \theta_{0} \wedge\left(d \theta_{0}\right)^{n}}, \quad \text { on } \partial D \text {. }
$$

We claim that

$$
\frac{\operatorname{det} H(\rho)}{J(\rho)}=\text { constant } \quad \text { on } D
$$

In fact, since $\log J(\rho)$ is pluriharmonic, by main theorem in [23] stated in Theorem 3.1, we have that $\operatorname{det} H(\rho) / J(\rho)$ attains its minimum over $\bar{D}$ at some point in $\partial D$. Thus, (3.8) holds for all $z \in \bar{D}$. Suppose that (3.9) fails, then there exist $z_{0} \in D$ and $\delta>0$ such that

$$
\frac{\operatorname{det} H(\rho)}{J(\rho)}>\frac{\int_{\partial D} \theta \wedge(d \theta)^{n}}{\int_{\partial D} \theta_{0} \wedge\left(d \theta_{0}\right)^{n}} \quad \text { in } B\left(z_{0}, \delta\right) .
$$


Let

$$
\theta_{s}=\frac{1}{2 i} \sum_{j=1}^{n+1}\left(\bar{z}^{j} d z^{j}-z^{j} d \bar{z}^{j}\right)
$$

Then, by Stokes' theorem,

$$
\int_{\partial D} \theta_{0} \wedge\left(d \theta_{0}\right)^{n}=\int_{D}\left(d \theta_{0}\right)^{n+1}=\int_{D} \operatorname{det} H\left(\rho_{0}\right)\left(d \theta_{s}\right)^{n+1}=\int_{D} J(\rho)\left(d \theta_{s}\right)^{n+1} .
$$

By the fact that (3.8) holds for all $z \in D$ and Stokes theorem

$$
\begin{aligned}
C & =\int_{\partial D} \theta \wedge(d \theta)^{n} \\
& =\int_{D}(d \theta)^{n+1} \\
& =\int_{D} \operatorname{det} H(\rho)\left(d \theta_{s}\right)^{n+1} \\
& =\int_{D} \frac{\operatorname{det} H(\rho)}{J(\rho)} J(\rho)\left(d \theta_{s}\right)^{n+1} \\
& >\frac{C}{\int_{\partial D} \theta_{0} \wedge\left(d \theta_{0}\right)^{n}} \int_{D} J(\rho)(z)\left(d \theta_{s}\right)^{n+1} \\
& =\frac{C}{\int_{\partial D} \theta_{0} \wedge\left(d \theta_{0}\right)^{n}} \int_{\partial D} \theta_{0} \wedge\left(d \theta_{0}\right)^{n} \\
& =C .
\end{aligned}
$$

This is a contradiction. Therefore,

$$
\frac{\operatorname{det} H(\rho)}{J(\rho)}=\text { constant }=\frac{\int_{\partial D} \theta \wedge(d \theta)^{n}}{\int_{\partial D} \theta_{0} \wedge\left(d \theta_{0}\right)^{n}} .
$$

By Theorem 3.1 and (3.13), there is a biholomorphic map $\phi$ from $D$ onto $B_{n+1}$, the unit ball in $\mathbb{C}^{n+1}$.

In addition, $J(\rho) \equiv 1$, then Theorem 3.1 and (3.13) imply that there is a biholomorphic map $\phi: D \rightarrow B_{n+1}$ so that $\operatorname{det} \phi^{\prime}(z) \equiv c$, a constant. This completes the proof of Theorem 1.2. 


\section{Acknowledgment}

Part of this work was done when the first author was visiting the Department of Mathematics at Chinese University of Hong Kong, whom he would like to thank for their hospitality. The second author is supported in part by RGC Hong Kong. Both authors thank the referee deeply for several very useful suggestions for this new vision of our paper, especially for the advice for a restatement of Theorem 1.2.

\section{References}

[1] S. M. Baouendi, P. Ebenfelt and L. P. Rothschild, Real Submanifolds in Complex Space and their Mappings, Math Series 47, Princeton University Press, Princeton, New Jersey, 1999.

[2] R. Beals, C. Fefferman and R. Grossman, Strictly pseudo-convex domains in $\mathbb{C}^{n}$, Bull. Amer. Math. Soc., (N.S.) 8 (1983), 125-322.

[3] D. Jr. Burns and S. Shnider, Spherical hypersurfaces in complex manifolds, Invent. Math. 33 (1976), 223-246.

[4] L. Caffarelli, J. J. Kohn, L. Nirenberg and J. Spruck, The Dirichlet problem for nonlinear second-order elliptic equations, II: complex MongeAmpère, and uniformly elliptic equations. Comm. Pure Appl. Math. 38 (1985), 209-252.

[5] D. C. Chang and S.-Y. Li, A Riemann zeta function associated to subLaplacian on the unit sphere in $\mathbb{C}^{n}$, J. d'Analyse Math. 86 (2002), $25-48$.

[6] S.-C. Chen and M.-C. Shaw, Partial Differential Equations in Several Complex Variables, Studies in Advanced Mathematics, ed. S.-T. Yau, AMS/International Press, 2000.

[7] S. Y. Cheng and S. T. Yau, On the existence of a complex Kähler metric on non-compact complex manifolds and the regularity of Fefferman's equation, Comm. Pure Appl. Math. 33 (1980), 507-544.

[8] S. S. Chern and S. Ji, On the Riemann mapping theorem, Ann. Math. 144 (1996), 421-439.

[9] C. Fefferman, Monge-Ampère equations, the Bergman kernel, and geometry of pseudo-convex domains, Ann. Math. 103 (1976), 395-416. 
[10] G. B. Folland and E. M. Stein, Estimates for $\bar{\partial}_{b}$-complex and analysis on Heisenberg group, Comm. Pure Appl. Math. 27 (1974), 429-522.

[11] N. Gamara, The CR Yamabe conjecture the case $n=1$, J. Eur. Math. Soc. 3 (2001), 105-137.

[12] N. Gamara and R. Yacoub, CR Yamabe conjecture - the conformally Flate case, Pacific J. Math. 201 (2001), 121-175.

[13] A. Greenleaf, The first eigenvalue of a sub-Laplacian on a pseudoconvex manifold, Comm. PDE 10(2) (1985), 191-217.

[14] X. Huang, On some problems in several complex variables and $C R$ geometry, First International Congress of Chinese Mathematicians, Beijing, 1998, 383-396.

[15] X. Huang and S. Ji, Global holomorphic extension of a local map and a Riemann mapping theorem for algebraic domains, Math. Res. Lett. 5 (1998), no. 1-2, 247-260.

[16] D. Jerison and J. Lee, The Yamabe problem on CR manifolds, J. Diff. Geom. 25 (1987), 167-197.

[17] D. Jerison and J. M. Lee, Extremals for the Sobolev inequality on the Heisenberg group and the CR Yamabe problem, J. Amer. Math. Soc. 1 (1988), no. 1, 1-13.

[18] D. Jerison and J. Lee, A subelliptic, nonlinear eigenvalue problem and scalar curvature on CR manifolds, Contemp. Math. 27 (1984), 57-63.

[19] S. G. Krantz, Function Theory of Several Complex Variables, 2nd edition, Wadsworth \& Brooks, 1992.

[20] J. M. Lee, Pseudo-Einstein structures on CR manifolds, Amer. J. Math. 110 (1988), no. 1, 157-178.

[21] J. M. Lee, The Fefferman metric and pseudo-Hermitian invariants, Trans. Amer. Math. Soc. 296 (1986), no. 1, 411-429.

[22] J. Lee and Melrose, Boundary behavior of the complex Monge-Ampère equation, Acta Math. (1982), 159-192.

[23] S.-Y. Li, Characterization for balls with potential function of KählerEinstein metrics for domains in $\mathbb{C}^{n}$, Commun. Anal. Geom. 13 (2005), 461-478. 
[24] S.-Y. Li, On the existence and regularity of Dirichlet problem for complex Monge-Ampère equations on weakly pseudo-convex domains, Calc. Var. PDE 20 (2004), 119-132.

[25] On the Neumann Problems of Complex Monge-Ampère equations, Indiana University J. of Mathematics, 44 (1995), 1099-1122.

[26] S.-Y. Li and H.-S. Luk, The Sharp lower bound for the first positive eigenvalues of sub-Laplacian on the pseudo-Hermitian manifold, Proc. AMS, 132 (2004), 789-798.

[27] H.-S. Luk, Affine connections and defining functions of real hypersurfaces in $C^{n}$, Trans. Amer. Math. Soc. 259 (1980), no. 2, 579-588.

[28] S. M. Webster, On the pseudo-conformal geometry of a Kähler manifold, Math. Z. 157 (1977), no. 3, 265-270.

[29] S. M. Webster, Pseudo-Hermitian geometry on a real hypersurface, J. Diff. Geom. 13 (1973), 25-41.

Department of Mathematics

UNIVERSITY OF CALIFORNIA

IRVINE

CA 92697-3875

USA

E-mail address: sli@math.uci.edu

Department of Mathematics

LADY ShaW BuILding

The Chinese University of Hong Kong

Shatin, N. T.

HONG KONG

E-mail address: hsluk@math.cuhk.edu.hk

Received September 5, 2005 
